\title{
Transverse profile and three-dimensional spin canting of a Majorana state in carbon nanotubes
}

\author{
Lars Milz, ${ }^{1}$ Wataru Izumida ${ }^{1,2}$ Milena Grifoni, ${ }^{1}$ and Magdalena Marganska $\odot^{1, *}$ \\ ${ }^{1}$ Institute for Theoretical Physics, University of Regensburg, 93053 Regensburg, Germany \\ ${ }^{2}$ Department of Physics, Tohoku University, Sendai 980 8578, Japan
}

(Received 11 April 2019; revised manuscript received 5 August 2019; published 16 October 2019)

\begin{abstract}
The full spatial 3D profile of Majorana bound states (MBS) in a nanowirelike setup featuring a semiconducting carbon nanotube (CNT) as the central element is discussed. By atomic tight-binding calculations, we show that the chiral nature of the CNT lattice is imprinted in the MBS wave function which has a helical structure, anisotropic in the transverse direction. The local spin-canting angle displays a similar spiral pattern, varying around the CNT circumference. We reconstruct the intricate 3D profile of the MBS wave function analytically, using an effective low-energy Hamiltonian accounting both for the electronic spin and valley degrees of freedom of the CNT. In our model, the four components of the Majorana spinor are related by the three symmetries of our Bogoliubov-de Gennes Hamiltonian, reducing the number of independent components to one. A Fourier transform analysis uncovers the presence of three contributions to the MBS, one from the $\Gamma$-point and one from each of the Fermi points, with further complexity added by the presence of two valley states in each contribution.
\end{abstract}

DOI: 10.1103/PhysRevB.100.155417

Over the past decade, Majorana fermions have been of great interest in condensed-matter physics. Under special conditions, they arise as quasiparticles in superconductors [1], where they are zero energy eigenstates of the Bogoliubov-de Gennes $(\mathrm{BdG})$ Hamiltonian and of the particle-hole symmetry operator. Theoretically, such quasiparticles were predicted to appear in the elusive one-dimensional $p$-wave superconductors [2] but it is also possible to engineer $s$-wave systems in such a way that they mimic $p$-wave superconductivity [3]. The most popular setup is based on semiconducting nanowires with large spin-orbit interaction and large $g$-factor in contact with a superconductor, which induces superconducting proximity correlations in the wire [4,5]. Although the experiments are by now very advanced [6], a definite proof that the reported signatures [7-10] are really due to the topologically nontrivial Majorana bound states (MBSs) is still missing. Thus, recent proposals have suggested to use local probes to infer exclusive properties of a MBS, such as its nonlocality and its peculiar spin canting structure [11-16], or the maximal electron-hole content of the Majorana spinor $[17,18]$. However, to exclude spurious effects, local experiments can be truly useful only if the spatial profile of the MBS is known with sufficient accuracy. This is very difficult to achieve for the case of the semiconducting nanowires, since their diameter of a few tens of nanometers and their length of several hundreds of nanometers do not allow for a microscopic calculation of the MBS wave function. Typically, the spatial profile is obtained with simple one-dimensional models [19]. The transverse profile has so far been obtained numerically for effective models: of core-shell nanowires in cylindrical $[20,21]$ and prismatic [22,23], and of full nanowires in hexagonal [24] geometries.

*Corresponding author: magdalena.marganska@ur.de
In this paper, we show that the spatial profile of MBSs can be derived analytically with good accuracy in a setup which uses a carbon nanotube (CNT) in proximity with an $s$-wave superconductor. Similar to the nanowires, such CNTs can host MBSs at their ends [25-29]. Due to their hollow character and small diameter, CNTs of several micrometers can be simulated numerically based on tight-binding models of carbon atoms on a rolled graphene lattice [30,31]. Such simulations allow one to accurately evaluate the excitation spectrum and local observables. Effective single-particle low energy models can be derived which well reproduce microscopic simulations [32].

In a recent paper [29], we used a four-band and an effective one-band model to calculate the topological phase diagram and the energy spectrum of proximitized semiconducting CNTs in perpendicular magnetic field, see Fig. 1(a), with parameters obtained from a fit to the numerical spectra [33].

In this paper, we use the same models to analytically obtain the full 3D spatial profile of the Majorana wave function. First, we exploit our knowledge of the three symmetries of the effective BdG Hamiltonian to derive the relations between the four components of the Majorana spinor [see Figs. 1(e) and 1(f)], thus reducing the number of independent components to one. Second, we find that the presence of two angular momentum contributions (valleys) and the spin degree of freedom results in the formation of a composite, six-piece MBS whose $3 \mathrm{D}$ wave function has a distinctive spiral pattern with a $C_{2}$ symmetry, impossible to factorize into separate transverse and longitudinal profiles. Equally nonisotropic is the spin canting angle, a quantity encoding the relative phase of the spin-up and spin-down particle components of the Majorana wave function. A comparison with the numerical results for the MBS of a $(12,4)$ CNT gives us confidence in the reliability of the effective model. Our results show that while simple 1D models can capture the important low-energy properties of the $\mathrm{BdG}$ spectrum, they might miss crucial features present in the 


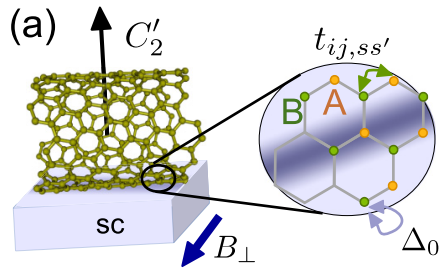

(c)

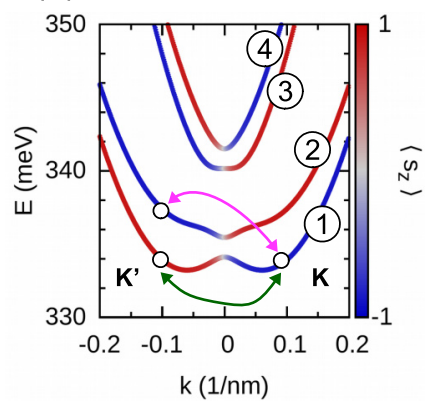

(e)

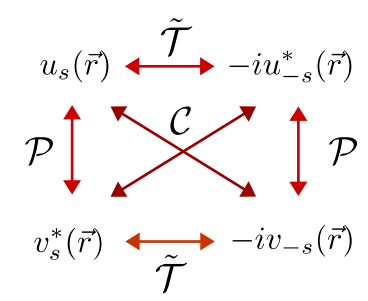

(b)

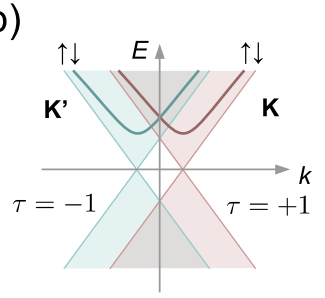

(d)

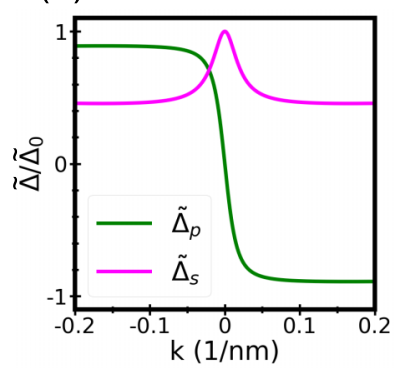

(f)

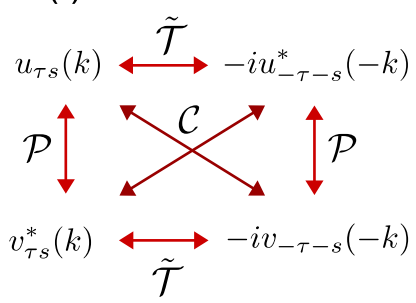

FIG. 1. Setup and bulk properties of a $(12,4)$ carbon nanotube with proximity-induced superconductivity. (a) Schematic of the system including the CNT which lies on top of an $s$-wave superconductor (SC) with a magnetic field applied perpendicular to the nanotube axis. The nearest-neighbor hopping $t_{i j, s s^{\prime}}$ is spin-dependent due to curvature. The superconducting substrate breaks the rotational symmetry of the nanotube with respect to the CNT axis, which induces a valley-mixing term in the Hamiltonian. Moreover, it generates an on-site superconducting pairing term $\Delta_{0}$. The numerical values of the various parameters of the model can be found in Appendix A 1 . (b) The low energy spectrum of the CNT consists of 1D cuts across the Dirac cones, with two valleys and two spin directions at each energy. (c) The single-particle energy spectrum of a $(12,4)$ nanotube in the vicinity of the $\Gamma$-point for a magnetic field of $B_{\perp}=14 \mathrm{~T}$. Color scale shows the expectation value of $\left\langle s_{z}\right\rangle$ for the corresponding energy state. A finite $\Delta_{0}$ induces in the $k$-space two superconducting pairing terms $\tilde{\Delta}_{s}(k)$ and $\tilde{\Delta}_{p}(k)$ whose action is indicated by the magenta and green lines, respectively. (d) The two superconducting pairing terms $\tilde{\Delta}_{s}(k)$ (interband), and $\tilde{\Delta}_{p}(k)$ (intraband), as functions of $k$. (e) The action of the particle-hole $\mathcal{P}$, pseudo-time-reversal $\tilde{\mathcal{T}}$ and chiral $\mathcal{C}$ operations on the components of a Nambu spinor in the real space. (f) The counterpart of these relations in the reciprocal space. The fact that $\mathcal{P}$ relates $u_{\tau s}(k)$ and $v_{\tau s}^{*}(k)$ follows from $\mathcal{P} \gamma_{k}=\gamma_{-k}^{\dagger}$.

full 3D wave function. This can have profound implications in various setups, where the shape and local spin composition of an MBS are relevant [12,15,16].

The paper has the following structure. In Sec. I, we discuss our microscopic model of the CNT, the symmetries of the $\mathrm{BdG}$ Hamiltonian in our setup, and the resulting relations

between the components of the Majorana spinor. In Sec. II, we show and discuss the numerical results of the spin canting of the full 3D MBS. We proceed to reconstruct the MBS analytically. First, we introduce in Sec. III the effective lowenergy model of the CNT, including the superconducting correlations. We also derive the form of the Majorana state in a continuum 1D approximation. In Sec. IV, we calculate the 3D Majorana solution and determine its full spatial profile. Finally, we compare the numerical results from the real-space tight-binding calculation with those of the analytical model.

\section{MODEL AND ITS SYMMETRIES}

Geometrically, a single wall CNT is equivalent to a rolledup strip taken from the two-dimensional honeycomb of carbon atoms that makes up a graphene sheet [34]. The band structure of the CNT can be obtained from that of graphene by imposing periodic boundary conditions in the transverse direction, which quantize the transverse momentum, turning the two-dimensional dispersion of graphene into a series of 1D cuts, which are the CNTs one-dimensional subbands, shown schematically in Fig. 1(b). Effective low-energy Hamiltonians can be derived from the microscopic model [32]. Thus, like in graphene, the low-energy band structure in nanotubes consists of two distinct and time-conjugate valleys $K$ and $K^{\prime}$ which are indexed by the quantum number $\tau(\tau=+1$ for $K$ valley and $\tau=-1$ for $K^{\prime}$ valley) [cf. Fig. 1(b)]. However, the simple fact of being rolled up drastically modifies the band structure, leading to effects that are not present in graphene. These are a curvature-induced band gap and an enhanced spin-orbit coupling [30-32,35]. The spin-orbit coupling in the nanotubes results in an effective spin-orbit field directed along the tube axis, with the sign of the field given by $\tau s$, with $s$ the spin quantum number along the CNT. The CNT's tiny diameter reduces the number of relevant transverse modes to exactly four in the low-energy regime, one for each spin and valley. We consider nanotubes of the zigzag class [36,37], where the Dirac points are only slightly shifted from $k=0$. Therefore the states near the $\Gamma$ point play a dominant role in the low energy physics. In order to open the gap at the $\Gamma$ point, we need to remove the Kramers degeneracy between the $(\tau, s)$ and $(-\tau,-s)$ states. The spin degeneracy can be removed by a transverse magnetic field, but only if the valleys are also mixed. Fortuitously, this happens automatically when the nanotube is in contact with the bulk superconductor, i.e., the source of the proximity effect. Its presence breaks the rotational symmetry of the tube, introducing mixing between the $K$ and $K^{\prime}$ valley. The resulting spectrum in a normal CNT is shown in Fig. 1(c). This effect will also be present even above the critical temperature, when the substrate is not superconducting. We model it in the same way as in Ref. [29], as a larger electrostatic potential for atomic sites in the neighborhood of the substrate.

As we will see, high magnetic fields are needed to induce the formation of the MBS. A suitable substrate which would withstand such fields is, e.g., $\mathrm{NbSe}_{2}$, where superconductivity has been shown to survive up to $30 \mathrm{~T}$ [38].

The proximity to a superconducting substrate induces Cooper pairing in the CNT. The excitation spectrum of the system can be determined from the BdG Hamiltonian, where 
the superconducting correlations are treated in a mean-field approximation. In our microscopic model, we assume an isotropic on-site pairing term [39], see Fig. 1(a). In a more realistic approach, the proximity effect arises from the Andreev reflection processes and it is strongest at the substrate/CNT interface. When we model this local proximity by assuming an exponential decay of the pairing away from the interface, our results, shown in Appendix D, do not differ qualitatively from the case of isotropic pairing. The analytical construction of an effective model with decaying proximity is, however, strongly complicated, therefore, in the following, we shall consider isotropic pairing. Using the Nambu spinor, we can construct the microscopic BdG Hamiltonian of our system. To anticipate the discussion in Sec. III, in the reciprocal space we obtain both an interband ( $\tilde{\Delta}_{s}$, with $s$-wave symmetry) and an in-band $\left(\tilde{\Delta}_{p}\right)$ pairing, with $p$-wave symmetry, required for topological superconductivity. The two pairings are shown in Fig. 1(d).

The CNT alone has a crystalline symmetry of rotation by $\pi$ around an axis perpendicular to the $\mathrm{CNT}\left[C_{2}^{\prime}\right.$ axis in Fig. 1(a)]. In consequence, the CNT on a superconducting substrate is a topological crystalline superconductor $[40,41]$ with the $C_{2}^{\prime}$ axis oriented as shown in Fig. 1(a). In our setup, however, the $C_{2}^{\prime}$ symmetry is broken by the magnetic field parallel to the substrate and only the local symmetries remain.

The true time-reversal symmetry is broken by the magnetic field. Nevertheless, the inspection of the single-particle Hamiltonian of our CNT setup in the real space used in our previous work —cf. Eq. (1) of Ref. [29]—with the lattice hoppings given by Eq. (9) in Ref. [42], shows that all its dominant terms are either spin independent and real or proportional to the spin and purely imaginary. This implies that our system possesses a local antiunitary symmetry, which involves a spin flip and commutes with the Hamiltonian. Its action is defined by $\tilde{\mathcal{T}} a c_{s}^{\dagger}(\vec{r})=-i a^{*} c_{-s}^{\dagger}(\vec{r})$, where $c_{s}^{\dagger}(\vec{r})$ is the operator creating an electron with spin $s$ at position $\vec{r}$. Contrary to the true time reversal, $\tilde{\mathcal{T}}$ has bosonic nature, $\tilde{\mathcal{T}}^{2}=1$. In our previous paper [29], we attributed $\tilde{\mathcal{T}}$ 's physical origin to the $C_{2}^{\prime}$ symmetry, but they are in fact unrelated $-\tilde{\mathcal{T}}$ is local while $C_{2}^{\prime}$ is not. The $\tilde{\mathcal{T}}$ is discussed further in the Appendix A 2 .

The second local symmetry is the particle-hole symmetry $\mathcal{P}$, inherent in all $\mathrm{BdG}$ systems. With the $\mathcal{P}$ and $\tilde{\mathcal{T}}$ symmetries combined, the BdG Hamiltonian of the nanotube is also chiral symmetric under $\mathcal{C}=\tilde{\mathcal{T}} \mathcal{P}$. When acting on the eigenstates of the finite system, expressed in the Nambu space as $\hat{\Psi}(\vec{r})=$ $\sum_{s}\left[u_{s}(\vec{r}) c_{s}(\vec{r})+v_{s}(\vec{r}) c_{s}^{\dagger}(\vec{r})\right]$, these operators convert between the $u_{s}$ and $v_{s}$ components of the different states in the way shown schematically in Fig. 1(e). (The $\tilde{\mathcal{T}}$ relation has been noticed in Ref. [15], although without attributing it to the presence of a pseudo-time-reversal symmetry.) The complementary relations holding in the reciprocal space, calculated in Sec. IV, are shown in Fig. 1(f). The presence of these three symmetries has a profound impact on the Majorana state.

The wave function of the MBS is given by $\left\langle\vec{r} \mid \Psi_{M}\right\rangle=$ $\Psi_{M}(\vec{r})$, where $\left|\Psi_{M}\right\rangle=\hat{\gamma}_{M}|0\rangle$ and $\hat{\gamma}_{M}^{\dagger}=\hat{\gamma}_{M}$ is the Majorana creation operator. Here $\vec{r}=\left(z, r_{\perp}\right)$, where $z$ and $r_{\perp}$ denote the longitudinal and the transverse components, respectively. The MBS is described by a spinor, $\Psi_{M}(\vec{r})=$ $\left(u_{M \uparrow}(\vec{r}), u_{M \downarrow}(\vec{r}), v_{M \uparrow}(\vec{r}), v_{M \downarrow}(\vec{r})\right)^{T}$, with $u_{M s}(\vec{r})$ and $v_{M s}(\vec{r})$ the electron and hole components, respectively, and $s$ indicating the spin degree of freedom. As detailed below, it is enough to find the $u_{M \uparrow}(\vec{r})$ components and use the symmetries of the underlying $\mathrm{BdG}$ Hamiltonian to determine the rest.

The first relation is a consequence of the fundamental property $\mathcal{P} \Psi_{M}(\vec{r}) \stackrel{!}{=} \Psi_{M}(\vec{r})$ of a Majorana state. Thus the relation $\mathcal{P} u_{s}(\vec{r})=v_{s}^{\star}(\vec{r})$ becomes $u_{M s}(\vec{r})=v_{M s}^{\star}(\vec{r})$. As we will show in Sec. III, the MBS are also eigenstates of the chiral symmetry $\mathcal{C}$, implying $v_{M s}(\vec{r})=i u_{M,-s}(\vec{r})$. Finally, since $\mathcal{C}=$ $\tilde{\mathcal{T}} \mathcal{P}$, the Majorana state must be an eigenstate of $\tilde{\mathcal{T}}$ as well, yielding the last relation $u_{M s}(\vec{r})=-i u_{M,-s}^{\star}(\vec{r})$. The relations illustrated in Fig. 1(e) and 1(f) become equalities within the Majorana spinor.

\section{SPIN CANTING OF THE MAJORANA STATE}

In the nanowire/quantum dot setups where the character of the potential MBS is determined by analyzing its coupling to the discrete levels of a quantum dot, the spin canting of the MBS turns out to play an important role $[12,15,16]$. If there is a mismatch between the spin of the MBS and that of the electron on the quantum dot, the coupling, and in consequence the conductance, is suppressed. Thus we turn next to examine the local spin canting angle in our Majorana nanotube.

We first notice that the total spin of the Majorana particle, summed over both particle and hole contributions, is zero. Thus, we focus on the relative spin composition of the particle components, $\left(u_{M \uparrow}, u_{M \downarrow}\right)$. These are complex quantities for the considered CNT setup. The local expectation value for each spin direction in the particle sector is given by $\left\langle\vec{u}_{M}(\vec{r})\left|s_{\alpha}\right| \vec{u}_{M}(\vec{r})\right\rangle$, where $s_{\alpha}$ are the Pauli matrices, $\alpha=x, y, z$, and $\vec{u}_{M}(\vec{r})=\left(u_{M \uparrow}(\vec{r}), u_{M \downarrow}(\vec{r})\right)^{T}$ is the electron component of the wave function.

Due to the symmetry relations, see Fig. 1(e) and Ref. [15], for the Majorana state it holds:

$$
\begin{aligned}
\left\langle\vec{u}_{M}(\vec{r})\left|s_{x}\right| \vec{u}_{M}(\vec{r})\right\rangle & =-2 \operatorname{Im}\left(u_{M \uparrow}^{2}(\vec{r})\right), \\
\left\langle\vec{u}_{M}(\vec{r})\left|s_{y}\right| \vec{u}_{M}(\vec{r})\right\rangle & =-2 \operatorname{Re}\left(u_{M \uparrow}^{2}(\vec{r})\right), \\
\left\langle\vec{u}_{M}(\vec{r})\left|s_{z}\right| \vec{u}_{M}(\vec{r})\right\rangle & =0 .
\end{aligned}
$$

The expectation value $\left\langle s_{z}\right\rangle$ is zero because of the pseudo-timereversal symmetry. Knowing the values of $\left\langle s_{x}(\vec{r})\right\rangle$ and $\left\langle s_{y}(\vec{r})\right\rangle$, we can define a local spin direction in the plane perpendicular to the nanotube:

$$
\theta_{x y}(\vec{r})=\arctan \left(\frac{\left\langle s_{y}(\vec{r})\right\rangle}{\left\langle s_{x}(\vec{r})\right\rangle}\right)=\pi / 2-2 \arg \left(u_{M \uparrow}(\vec{r})\right) .
$$

The full 3D spatial profile of the wave function together with the local $\theta_{x y}(\vec{r})$ for our numerically obtained Majorana state is shown in Fig. 2(a). The distance from the CNT surface encodes the local amplitude of the MBS wave function, $\left|u_{M \uparrow}(\vec{r})\right|$, and the color scale maps $\theta_{x y}(\vec{r})$. The oscillation of $\theta_{x y}$ along $z$ with the same period as the MBS wave function is clearly visible. Further, Fig. 2(b) shows a zoom of the left end of the tube for the first peak of $\left|u_{M \uparrow}(\vec{r})\right|$ along $z$, polar angle $\varphi$ resolved and displaying the helical pattern of $\theta_{x y}$. Finally, Fig. 2(c) visualizes the local spin canting at the very left end of the nanotube, where the electron tunneling would occur. The spin-canting angle takes several different values at the edge atoms, with visible $C_{2}$ symmetry. Thus the tunneling from 


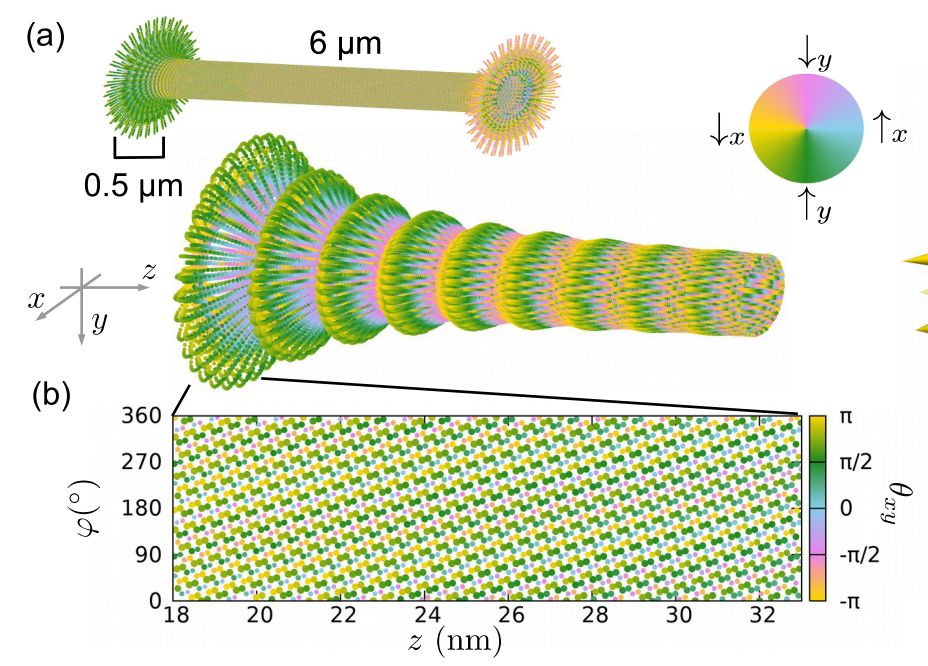

(c)

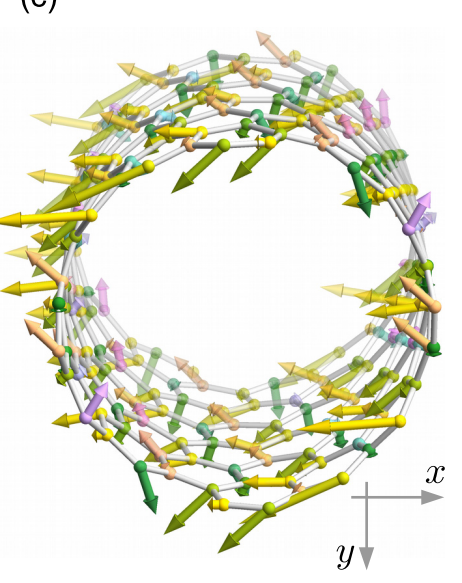

FIG. 2. Spin-canting angle $\theta_{x y}(\vec{r})$ and the amplitude $\left|u_{M \uparrow}(\mathbf{r})\right|$ of the electronic component of the Majorana state, obtained in a real-space tight-binding calculation of a finite $(12,4) \mathrm{CNT}$ with 4000 unit cells $(L=6.14 \mu \mathrm{m})$ for a magnetic field $B_{\perp}=14 \mathrm{~T}$. In all panels, the color corresponds to the local value of $\left\langle\theta_{x y}\right\rangle$. (a) The full Majorana state and its leftmost $0.5 \mu \mathrm{m}$, with distance from the CNT surface encoding $\left|u_{\uparrow}(\mathbf{r})\right|$. (b) $2 \mathrm{D}$ projection of the region with the first maximum of the Majorana wave function, with point size corresponding to $\left|u_{M \uparrow}(\mathbf{r})\right|$. (c) The left termination (i.e., the first $1.8 \mathrm{~nm}$ ) of the CNT lattice. Vector length corresponds to $\left|u_{M \uparrow}(\mathbf{r})\right|$, its orientation to the spin canting angle. In both (b) and (c), note the variation of $\left\langle\theta_{x y}\right\rangle$ with the polar coordinate.

a putative quantum dot coupled to the left end is definitely different than in a nanowire, where the spin is assumed to have only one direction on the whole nanowire cross section. A full blockade of one spin direction of the left Majorana state [15] could never be achieved in a nanotube. In shorter systems, like the nanowires studied in Refs. [12,16], the quantum dot would always (in the language of the authors) be coupled both to the left and right Majorana states. Whether this effect is helpful or detrimental for the experiment is not yet clear and will be the object of our future work.

The full spin texture could be directly resolved only by local measurements, for example, with a spin-polarized STM tip [43].

\section{EFFECTIVE FOUR AND ONE-BAND MODEL}

The low-energy Hamiltonian of a nonsuperconducting CNT in the basis $\left\{|k K \uparrow\rangle,|k K \downarrow\rangle,\left|k K^{\prime} \uparrow\right\rangle,\left|k K^{\prime} \downarrow\right\rangle\right\}$ is given by

$$
H(k)=\left(\begin{array}{cccc}
\xi_{K \uparrow}(k) & \mu_{B} B_{\perp} & \Delta_{K K^{\prime}} & 0 \\
\mu_{B} B_{\perp} & \xi_{K \downarrow}(k) & 0 & \Delta_{K K^{\prime}} \\
\Delta_{K K^{\prime}} & 0 & \xi_{K^{\prime} \uparrow}(k) & \mu_{B} B_{\perp} \\
0 & \Delta_{K K^{\prime}} & \mu_{B} B_{\perp} & \xi_{K^{\prime} \downarrow}(k)
\end{array}\right),
$$

where $\xi_{\tau s}(k)=\varepsilon_{\tau s}(k)-\mu$ is the single-particle energy measured with respect to the chemical potential $\mu, \varepsilon_{\tau s}(k)$ is the single-particle energy of the electrons [see Eq. (A4)], $\Delta_{K K^{\prime}}$ is the energy scale associated with the valley mixing (in our calculation, it is $\sim 2.5 \mathrm{meV}$ ) and $\mu_{B} B_{\perp}$ is the Zeeman energy due to the perpendicular magnetic field $B_{\perp}$. Diagonalization of this Hamiltonian results in four spin- and valley-mixed bands shown in Fig. 1(c). We can safely neglect any contributions from disorder, because CNTs can be grown with ultraclean lattices [44-46]. The Bloch Hamiltonian can be solved analytically with the assumption that the correlation induced by the magnetic field between lower (1), (2) and the upper (3), (4) pairs of bands is negligible [29]. When the chemical potential is set in the lower gap at the $\Gamma$ point, this approximation allows us to consider only the lower bands $\tilde{E}_{1}(k)$ and $\tilde{E}_{2}(k)$; it holds for $\mu_{B} B_{\perp}$ smaller than both of the spin-orbit coupling and the valley mixing energy scales, which in our case are $\sim 2 \mathrm{meV}$. The details of the calculation and a short discussion of the CNT properties are presented in the Appendix A 1.

In the eigenbasis of Eq. (2) with the two-band approximation, the corresponding BdG Hamiltonian for our system is given by

$$
\tilde{\mathcal{H}}_{\mathrm{BdG}}=\left(\begin{array}{cccc}
\tilde{E}_{1}(k) & 0 & \tilde{\Delta}_{p}(k) & -\tilde{\Delta}_{s}(k) \\
0 & \tilde{E}_{2}(k) & \tilde{\Delta}_{s}(k) & \tilde{\Delta}_{p}(k) \\
\tilde{\Delta}_{p}(k) & \tilde{\Delta}_{s}(k) & -\tilde{E}_{1}(k) & 0 \\
-\tilde{\Delta}_{s}(k) & \tilde{\Delta}_{p}(k) & 0 & -\tilde{E}_{2}(k)
\end{array}\right) .
$$

Out of the two superconducting pairing terms, $\tilde{\Delta}_{s}(k)=$ $\tilde{\Delta}_{s}(-k)$ is an even function of $k$, while $\tilde{\Delta}_{p}(k)=-\tilde{\Delta}_{p}(-k)$ is an odd function of $k$, see Fig. 1(d). The pairing term $\tilde{\Delta}_{p}(k)$ can be viewed as a $p$-wave like gap. The BdG Hamiltonian Eq. (3) can be partly diagonalized, taking into account the blocks with the single-particle energies $\tilde{E}_{1}(k), \tilde{E}_{2}(k)$ and the superconducting gap $\tilde{\Delta}_{s}(k)$. Details of this calculation are given in Appendix A3. Then, the rotated BdG Hamiltonian is block diagonal and the blocks are given by

$$
\hat{\mathcal{H}}_{\mathrm{BdG}}^{ \pm}=\left(\begin{array}{cc}
\tilde{\xi}_{ \pm}(k) & \tilde{\Delta}_{p}(k) \\
\tilde{\Delta}_{p}(k) & -\tilde{\xi}_{ \pm}(k)
\end{array}\right) .
$$

The quasiparticle energies $\tilde{\xi}_{ \pm}(k)$ are

$$
\begin{aligned}
\tilde{\xi}_{ \pm}(k)= & \frac{1}{2}\left(\tilde{E}_{1}(k)-\tilde{E}_{2}(k)\right) \\
& \pm \frac{1}{2} \sqrt{\left(\tilde{E}_{1}(k)+\tilde{E}_{2}(k)\right)^{2}+4 \tilde{\Delta}_{s}^{2}(k) .}
\end{aligned}
$$

The functions $\tilde{\xi}_{+}(k)$ and $\tilde{\Delta}_{p}(k)$ are sketched in Fig. 3(a). The low-energy physics, relevant for the Majorana states, is 
(a)

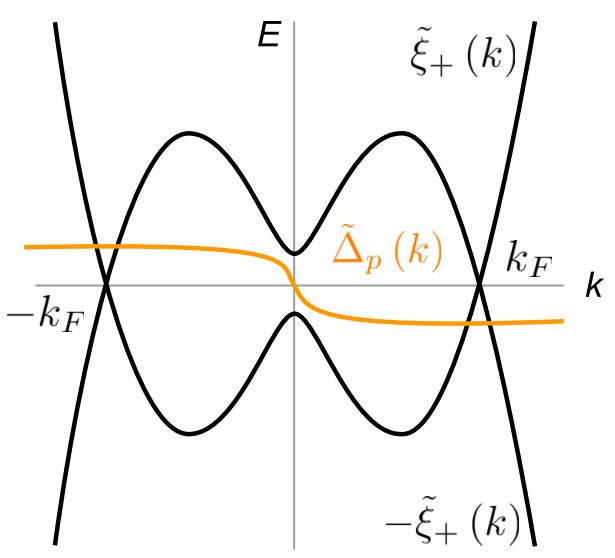

(b)

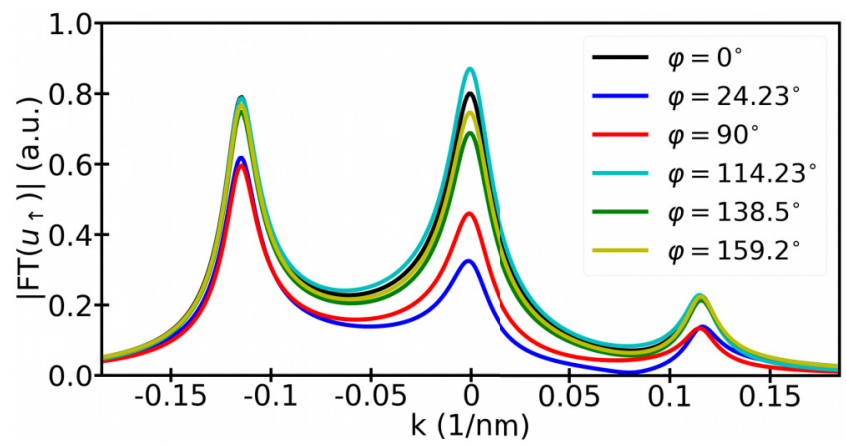

FIG. 3. $k$-space properties of a proximitized CNT in magnetic field at low energies. (a) Quasiparticle energy $\tilde{\xi}_{+}(k)$ and superconducting order parameter $\tilde{\Delta}_{p}(k)$ in the effective one-band model. The superconducting order paramater is an odd function of the momentum $k$. Three $k$ values generate the dominant contributions to zero energy modes: one comes from the $\Gamma$ point and one from each of the Fermi points, $\pm k_{F}$. (b) The Fourier transform of the numerical Majorana wave function for different azimuthal cuts $\varphi$ confirms that the zero mode contains only three dominant $k$ contributions.

described by the block $\hat{\mathcal{H}}_{\mathrm{BdG}}^{+}$. The particle-hole symmetry operator for the $\hat{\mathcal{H}}_{\mathrm{BdG}}^{+}$block is $\mathcal{P}=\tau_{x} \mathcal{K}$, and the chiral symmetry operator is $\mathcal{C}=\tau_{y}$, where $\tau_{x, y, z}$ are the Pauli matrices acting in the two-dimensional subspace of each block.

\section{ANALYTICAL RECONSTRUCTION OF THE 3D MBS WAVE FUNCTION}

\section{A. 1D Majorana profile}

MBSs are zero-energy eigenstates of the BdG Hamiltonian and of the particle-hole symmetry operator. From the behavior of $\tilde{\xi}_{+}(k)$, we infer that the low-energy physics has three contributions: one from the $\Gamma$ point and one from each of the Fermi points. This ansatz is confirmed by the Fourier transforms for several azimuthal cuts $\left(\varphi=r_{\perp} / R=\right.$ const $)$ of the numerically obtained MBS wave function, shown in Fig. 3(b). One clearly sees one peak at the $\Gamma$ point and two peaks at opposite momenta. The peak locations are independent of $\varphi$ but their height is not. Furthermore, the peak at negative $k$ is larger. This is caused by the helical spin structure of the single-particle spectrum, shown in Fig. 1(b). The solution at $\pm k_{F}$ is generated mostly by the band (1), and spin $\uparrow$ for this band is associated with $k<0$.
Thus, similar to some 1D models for nanowires [19], the generic form of a Majorana state can be defined as

$$
\left|\Psi_{M}\right\rangle=\frac{A_{\Gamma}}{\sqrt{2}}\left|\Psi_{\Gamma}\right\rangle+\frac{A_{R}}{\sqrt{2}}\left|\Psi_{k_{F}}\right\rangle+\frac{A_{L}}{\sqrt{2}}\left|\Psi_{-k_{F}}\right\rangle .
$$

We will later take into account the 3D nature of each of these three contributions and reconstruct the 3D spatial profile of the Majorana wave function. For now, we approximate $\hat{\mathcal{H}}_{\mathrm{BdG}}^{+} \approx$ $\hat{\mathcal{H}}_{\mathrm{BdG}}^{\Gamma}+\hat{\mathcal{H}}_{\mathrm{BdG}}^{R}+\hat{\mathcal{H}}_{\mathrm{BdG}}^{L}$, where we make Taylor expansions around the momenta $k=0$ and $k= \pm k_{F}$, with $k_{F}$ determined by the constraint $\tilde{\xi}_{+}\left(k_{F}\right)=0$. The details of the calculation are presented in Appendix B.

Crucially, the spinorial components of the solutions at each of the three $k$ points are the same, which allows us to combine them into a single state which is also an eigenstate of both $\mathcal{P}$ and $\mathcal{C}$. With the three contributions we can construct the 1D solution from the generic solution Eq. (5). It is characterized by an exponential decay governed by the imaginary wave vectors $\kappa_{i}(i=\Gamma, L, R)$. The coefficients can be determined by the three constraints:

$$
\begin{aligned}
& \mathcal{P} \Psi_{M}(z) \stackrel{!}{=} \Psi_{M}(z), \\
& \Psi_{M}(z=0) \stackrel{!}{=} 0, \\
& \int_{0}^{\infty} d z\left|\Psi_{M}(z)\right|^{2} \stackrel{!}{=} 1 .
\end{aligned}
$$

From previous findings [29], we know that in the topological regime $\kappa_{\Gamma} \in \mathbb{R}$ and $\kappa_{R}, \kappa_{L} \in \mathbb{C}$. Moreover, it holds that $\operatorname{Re}\left(\kappa_{R}\right)=\operatorname{Re}\left(\kappa_{L}\right)$ and $\operatorname{Im}\left(\kappa_{R}\right)=-\operatorname{Im}\left(\kappa_{L}\right) \Leftrightarrow \kappa_{R}=\kappa_{L}^{\star}$. Therefore, the wave function can be written as

$$
\Psi_{M, 1 D}(z)=\left[\frac{A_{\Gamma}}{\sqrt{2}} e^{\kappa_{\Gamma} z}+\frac{A_{R}}{\sqrt{2}} e^{\kappa_{R} z}+\frac{A_{L}}{\sqrt{2}} e^{\kappa_{R}^{*} z}\right]\left(\begin{array}{c}
\mp i \\
1
\end{array}\right) .
$$

These eigenvectors are not eigenstates of the particle-hole operator $\mathcal{P}=\tau_{x} \mathcal{K}$, but we can multiply them by a complex number $c_{ \pm}= \pm 1+i$, such that they satisfy the Majorana constraint. Then, by applying the Majorana Eq. (6a) and the boundary Eq. (6b) conditions we get the 1D solution, which is given by

$$
\Psi_{M, 1 \mathrm{D}}(z)=\frac{\mathcal{N}}{2}\left(\psi_{\|}(z)+\psi_{\|}^{\star}(z)\right) \frac{1}{\sqrt{2}}\left(\begin{array}{c}
1-i \\
1+i
\end{array}\right),
$$

where

$$
\psi_{\|}(z)=\left(e^{\kappa_{F} z+i k_{F} z}-e^{\kappa_{\Gamma} z}\right)
$$

encodes the dependence of the wave function on the longitudinal coordinate. The sum $\psi_{\|}(z)+\psi_{\|}^{*}(z)$ satisfies the boundary condition Eq. (6b), and $\mathcal{N}$ is the normalization constant determined from Eq. (6c). The contribution from the $\Gamma$ point is a pure evanescent state and from the contribution from the Fermi points we get a decaying oscillation with the wave vector $k_{F}$.

\section{B. Reconstructing the 3D profile}

In the remaining part of this paper, we will provide the analytical form only for $u_{\uparrow}(\vec{r})$ (dropping the $M$ subscript for compactness of notation), since the remaining Majorana spinor components can be obtained by the application of $\mathcal{P}$, $\tilde{\mathcal{T}}$, and $\mathcal{C}$ symmetries. 
The Majorana operator to create the state Eq. (5) is defined as

$$
\hat{\gamma}_{M}=\sum_{k}\left(\begin{array}{l}
u \\
v
\end{array}\right)^{T}\left(\begin{array}{c}
d_{k+} \\
d_{-k+}^{\dagger}
\end{array}\right)
$$

where $k \in\left\{\Gamma, k_{F},-k_{F}\right\}$ and $u=v^{\star}=\frac{1-i}{\sqrt{2}}$. To find the analytical wave function, we need to transform the wave function from the one-band back to the four-band model; this procedure is discussed in Appendix C. To express the Majorana state in the sublattice- and spin-resolved basis, we need the transformations reversing Eqs. (A7), (A12), and (A18). At the end, we obtain

$$
\hat{\gamma}_{M}=\sum_{k, \tau, s}\left(u_{\tau s}(k) c_{k \tau s}+v_{\tau s}(-k) c_{-k \tau s}^{\dagger}\right),
$$

for $k \in\left\{\Gamma, \pm k_{F}\right\}$, where the coefficients $u_{\tau s}(k)$ correspond to the electron and $v_{\tau s}(k)$ to the hole contribution, respectively. We find a compact form for the coefficients,

$$
\begin{aligned}
& u_{\tau s}(k)=\tau s \Lambda_{\tau s}(k) \lambda_{s}(k), \\
& v_{\tau s}(k)=\tau s \Lambda_{\tau s}(k) \lambda_{s}^{\star}(k),
\end{aligned}
$$

with

$$
\Lambda_{\tau s}(k)= \begin{cases}a_{s}(k) & \text { for } \tau=+1 \\ b_{s}(k) & \text { for } \tau=-1\end{cases}
$$

and [see Eqs. (A8) for $a_{s}(k)$ and $\left.b_{s}(k)\right]$

$$
\lambda_{s}(k)=u m(k) g(s k)-s v n(k) h(s k) .
$$

The coefficients $g(k), h(k)$, and $n(k), m(k)$ are found below, in Eqs. (A13) and (A19), respectively. By using the relations $a_{s}(-k)=b_{-s}(k), g(-k)=h(k), m(-k)=m(k)$, $n(-k)=n(k)$, we obtain $\Lambda_{\tau s}(k)=\Lambda_{-\tau-s}(-k)$ and $\lambda_{s}(k)=$ $-i \lambda_{-s}^{\star}(-k)$. Finally, we arrive at the symmetry relations of the electron and hole coefficients $u_{\tau s}(k)$ and $v_{\tau s}(k)$ illustrated in Fig. 1(f).

We have now the expression of the wave function in conduction basis. To apply the boundary condition it must, however, be recast in the sublattice-resolved basis. In general, for the transformation into the sublattice basis one needs also the valence band contribution. Here we can use the fact that, due to the high chemical potential, we are far away from the charge neutrality point and therefore the contribution from the valence band is negligible. With this, the components in the sublattice basis are defined as $u_{p \tau s}(k)=e^{i p \eta_{\tau s k}} u_{\tau s}(k)$, where $\eta_{\tau s}(k)=\arg \left(\gamma_{\tau s}(k)\right)$ is the phase of Eq. (A2) in the low-energy regime, and $p=+1$ for $A$ sublattice and $p=-1$ for $B$ sublattice.

Since our nanotube is chiral, the open boundary conditions imply that the wave function must vanish on one end at the missing $A$ atoms and on the other end at the missing $B$ atoms [42]. We use therefore the open boundary condition $\Psi_{A}\left(z=0, r_{\perp}\right) \stackrel{!}{=} 0 \forall r_{\perp}$. The wave function $u_{p \uparrow}(\vec{r})$ is given by the superposition of the three contributions $k \in\left\{\Gamma, k_{F},-k_{F}\right\}$ and the two valleys $K$ and $K^{\prime}$, each with its specific transverse profile $e^{i \tau k_{\perp} x_{\perp}}$ :

$$
\begin{aligned}
& u_{p \uparrow}(\mathbf{r})=\frac{A_{\Gamma}}{\sqrt{2}}\left[e^{i p \eta_{K \uparrow \Gamma}} u_{K \uparrow}(\Gamma) e^{i k_{\perp} r_{\perp}}\right. \\
& \left.+e^{i p \eta_{K^{\prime} \uparrow \Gamma}} u_{K^{\prime} \uparrow}(\Gamma) e^{-i k_{\perp} r_{\perp}}\right] e^{\kappa_{\Gamma} z} \\
& +\frac{A_{R}}{\sqrt{2}}\left[e^{i p \eta_{K \uparrow k_{F}}} u_{K \uparrow}\left(k_{F}\right) e^{i k_{\perp} r_{\perp}}\right. \\
& \left.+e^{i p \eta_{K^{\prime} \uparrow k_{F}}} u_{K^{\prime} \uparrow}\left(k_{F}\right) e^{-i k_{\perp} r_{\perp}}\right] e^{\kappa_{F} z+i k_{F} z} \\
& +\frac{A_{L}}{\sqrt{2}}\left[e^{i p \eta_{K \uparrow-k_{F}}} u_{K \uparrow}\left(-k_{F}\right) e^{i k_{\perp} r_{\perp}}\right. \\
& \left.+e^{i p \eta_{K^{\prime} \uparrow-k_{F}}} u_{K^{\prime} \uparrow}\left(-k_{F}\right) e^{-i k_{\perp} r_{\perp}}\right] e^{\kappa_{F} z-i k_{F} z} .
\end{aligned}
$$

The amplitudes can be fixed by observing that the Majorana condition requires $A_{\Gamma} \in \mathbb{R}$ and $A_{R}=A_{L}^{\star}$. From the open boundary condition in the longitudinal direction, we obtain a relation between $A_{R}$ and $A_{\Gamma}$; hence, the particle component of the wave function can be written as

$$
\begin{aligned}
u_{p \uparrow}(\vec{r})= & \frac{1}{\sqrt{2}} \sum_{\tau} e^{i \tau k_{\perp} r_{\perp}}\left[A_{R} e^{i p \eta_{\tau \uparrow k_{F}}} u_{\tau \uparrow}\left(k_{F}\right) \psi_{\|}(z)\right. \\
& \left.+A_{R}^{\star} e^{i p \eta_{\tau \uparrow-k_{F}}} u_{\tau \uparrow}\left(-k_{F}\right) \psi_{\|}^{\star}(z)\right] .
\end{aligned}
$$

The expressions for $u_{\tau s}(k)$ are given in Eq. (10), and for $\psi_{\|}(z)$ in Eq. (8). The spatial profile of the wave function is not trivial, in the sense that it cannot be factorized into separate longitudinal and transverse profiles, $u_{p \uparrow}(\vec{r}) \neq f\left(r_{\perp}\right) g(z)$. The absolute value $\left|A_{R}\right|$ is fixed by the normalization and its phase by the Majorana condition. Note that the transverse momentum $k_{\perp}$ is quantized by the periodic boundary condition. The Fermi wave vector $k_{F}$ is given by the position of the chemical potential $\mu$, and the characteristic decay lengths at $\Gamma$ and $\pm k_{F}$ by the parameters of the Hamiltonian at this $\mu$. Thus all factors in the wave function are in principle known from the analytics.

\section{Comparison between analytical and numerical results}

To test the accuracy of our formula Eq. (13), we have performed a comparison between the analytical and numerical solutions for several 1D cuts of the full MBS profile, at varying values of the azimuthal angle $\varphi$. We fitted the numerical solutions with Eq. (13), finding for each cut the parameters $\kappa_{\Gamma}, \kappa_{F}, k_{F}$, and $A_{R}$.

The results for three values of the polar angle, $\varphi=$ $0^{\circ}, 24.23^{\circ}, 114.23^{\circ}$ are shown in Fig. 4 . The analytical model clearly reproduces very well the numerically obtained wave functions. However, due to the simplifications inherent in the effective one-band model, there are three aspects where we have to adjust for the lost information.

(i) In the microscopic model, the $\mathcal{P}$ symmetry holds exactly (by construction), but $\tilde{\mathcal{T}}$ is minimally broken by two small effects. One is the presence of the weak spin-flip terms in the Hamiltonian, due to the enhanced spin-orbit coupling $[30,32,42]$. The other is the small Peierls phase for the nearestneighbor hopping due to the magnetic field [47]. Thus in the numerical solution, the $\tilde{\mathcal{T}}$ - and $\mathcal{C}$-related components of the Nambu spinor differ by about $\pm 3 \%$. Removing the spin flip and the Peierls phase restores the $\tilde{\mathcal{T}}$ and consequently also the $\mathcal{C}$ symmetries, see Appendix A 2 for details. 

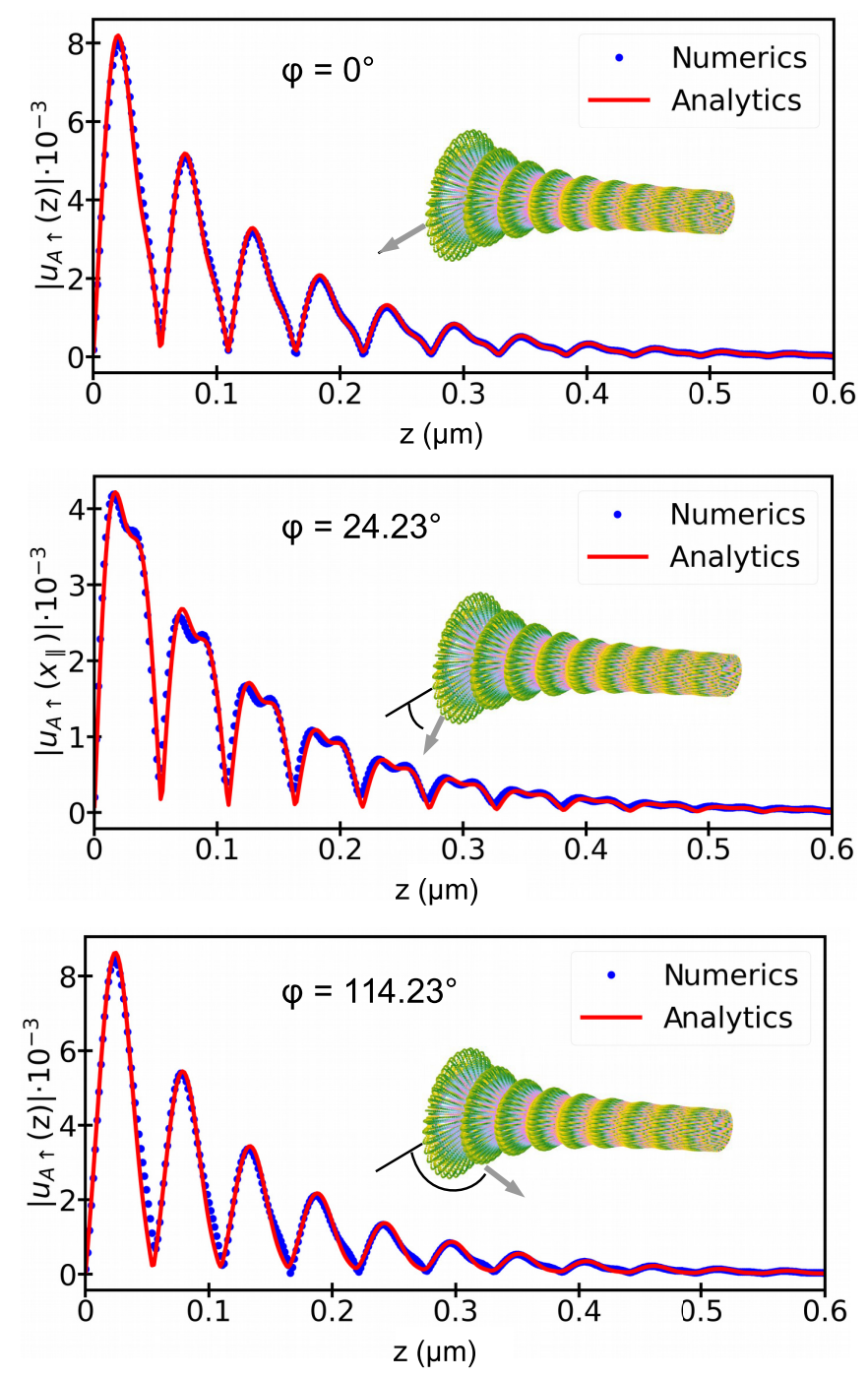

FIG. 4. Azimuthal cuts of the electron component $u_{A \uparrow}$ of the Majorana spinor for (a) $\varphi=0^{\circ}$, (b) $\varphi=24.23^{\circ}$, and (c) $\varphi=114.23^{\circ}$. The position of the cut in the full MBS wave function is indicated in each inset. The analytical form of $u_{\uparrow}$ is given by Eq. (13), its parameters are obtained from fits to the modulus of the numerical solution.

(ii) In the analytics, we neglected some correlations due to the magnetic field. Further, we performed Taylor expansions around the three momenta $k=0, \pm k_{F}$. Thus, the values $\kappa_{\Gamma}$, $\kappa_{F}$, and $k_{F}$ from the analytics are slightly different from those which are obtained by fitting the numerical data using Eq. (13), see Table I.

(iii) We implemented the valley mixing through a continuous potential ridge along the $\mathrm{CNT} /$ superconductor interface.

TABLE I. Values of $k_{F}, \kappa_{\Gamma}$, and $\kappa_{F}$ from the analytical calculation compared with values fitted from the numerics.

\begin{tabular}{lcc}
\hline \hline & Analytics $\left(\frac{1}{\mu m}\right)$ & Fits $\left(\frac{1}{\mu m}\right)$ \\
\hline$\kappa_{\Gamma}$ & -7.94 & -8.93 \\
$\kappa_{F}$ & -6.56 & -8.01 \\
$k_{F}$ & 118.92 & 115.25 \\
\hline \hline
\end{tabular}

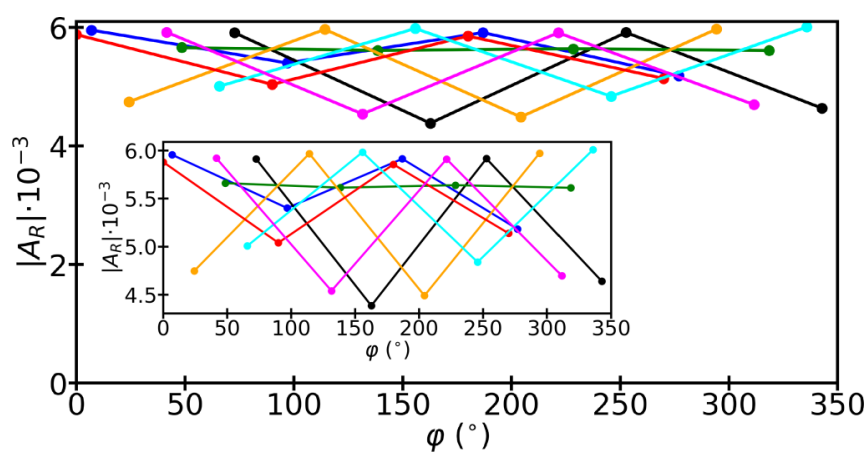

FIG. 5. The absolute value of the fitted amplitude $\left|A_{R}\right|$ of 28 different $\varphi$ cuts. The colors correspond to different groups of atoms related by the $C_{4}$ symmetry (i.e., atoms at the same $z$ position). From the inset, we see the approximate $\pi$ periodicity of $A_{R}$ and thus the $C_{2}$ symmetry of the MBS wave function.

This results in the coupling between the two valleys, but also in their coupling to higher transverse momentum bands which therefore also contribute, albeit very weakly, to the final Majorana state. In consequence, although we expect $A_{R}$ to be independent of $\varphi$, we obtain from the fitting procedure different $A_{R}$ for different $\varphi$ cuts, with the resulting values of $\left|A_{R}(\varphi)\right|$ shown in Fig. 5. We see that, although not constant, the amplitude $A_{R}$ is a weakly varying function of $\varphi$. Moreover, the data resolved for atoms at the same $z$ position show that $A_{R}$ is close to $\pi$ periodic. This is a consequence of the $C_{4}$ symmetry of our $(12,4) \mathrm{CNT}$ where the $K^{\prime} / K$ valley states carry the angular momentum $\ell= \pm 1$. Since the Majorana state is constructed predominantly from electron (and hole) states with $\ell= \pm 1$, the amplitude of its wave function, to which $A_{R}$ was fitted, has an approximate $C_{2}$ symmetry. This is also visible in Fig. 2(c), where the $C_{2}$ (instead of $C_{1}$ ) symmetry of spin texture arises from the factor of 2 in Eq. (1).

In Fig. 6, we show a comparison between the analytical and numerical results for $\operatorname{Re}\left(u_{A \uparrow}\right), \operatorname{Im}\left(u_{A \uparrow}\right)$ and the resulting canting angle $\theta_{x y}(z)$ for $\varphi=0$. The slight discrepancy between the numerical and analytical values of the real and imaginary parts of $u_{\uparrow}(\vec{r})$, shown in Figs. 6(a) and 6(b), is amplified in the spin-canting-angle behavior shown in Fig. 6(c). In particular, additional phase jumps are visible at positions where the real value in numerics is small and positive, while the analytical result is also small but negative. Nevertheless, the overall agreement is again good.

\section{CONCLUSIONS AND OUTLOOK}

In this paper, we have shown in a combination of numerical modeling and analytical calculations how to determine the full spatial profile of the MBS in a proximitized semiconducting CNT. The wave function has three contributions: one from the $\Gamma$ point and one from each Fermi point, which is also supported by an analysis of the numerical data via a Fourier transformation. We find the symmetry relations which must be fulfilled by the components of the Majorana spinor. The excellent agreement between the analytically obtained and the numerically calculated spin- and sublattice-resolved spinor gives us confidence in the accuracy of the local observables further derived in this paper. Despite being obtained for a 
(a)

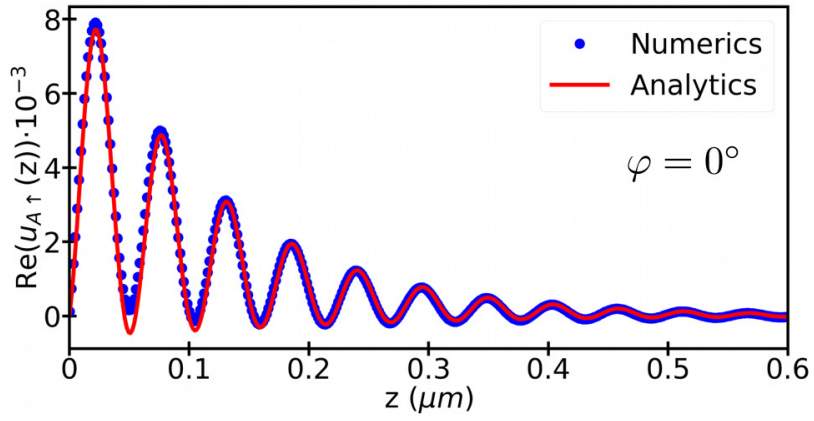

(b)

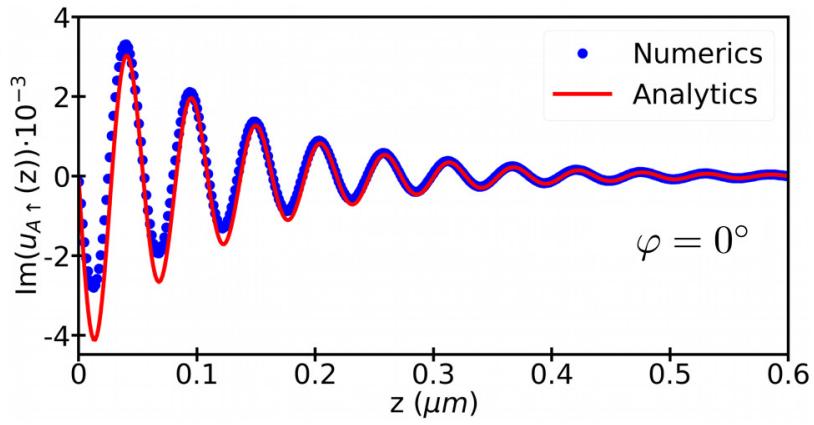

(c)

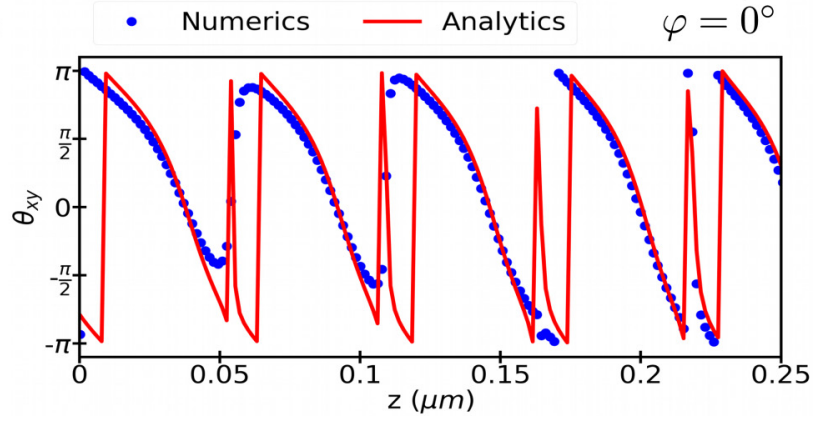

FIG. 6. (a), (b) Numerical and analytical $\operatorname{Re}\left(u_{A \uparrow}(z)\right)$ and $\operatorname{Im}\left(u_{A \uparrow}(z)\right)$ for the polar angle $\varphi=0^{\circ}$ with parameters from the $\left|u_{A \uparrow}(z)\right|$ fit. (c) The spin-canting angle $\theta_{x y}$, defined in Eq. (1), for the cut $\varphi=0^{\circ}$.

CNT, our results might also serve as a reference for other systems where a microscopic calculation of the MBS spinor is not possible. The features which our model captures very well are the three main momentum contributions to the MBS, the decaying behavior of the wave function combined with its spiral pattern, its oscillation, and the symmetries linking the different components of the Nambu spinor. We show that our analytical model fits very well the numerical data of the wave function obtained by a tight-binding calculation. Our results will be useful for modeling and interpreting the experimental results in a realistic quantum transport setup where the properties of the Majorana states are probed locally.

In our future investigations, we will study in detail the interactions between the substrate and the nanotube-both with respect to the tunneling processes which induce the proximity effect as well as the implications of high electric fields which may arise at the interface between the CNT and the substrate as a result of the charge transfer.

The code used to find the energy spectrum and eigenstates, as well as the data sets used in this paper are publicly available [48].

\section{ACKNOWLEDGMENTS}

The authors thank the Deutsche Forschungsgemeinschaft for financial support via the GRK 1570 and SFB 1277 (project B04) grants and the Bayerisches Staatsministerium für Wissenschaft und Kunst for funding within the IGK "Topological insulators" program of Elitenetzwerk Bayern, as well as the Japan Society for the Promotion of Science for the KAKENHI Grants (No. JP15K05118, No. JP15KK0147, and No. JP18H04282).

\section{APPENDIX A: CNT SPECTRUM}

\section{Single-particle spectrum}

The simplest way of obtaining the Hamiltonian of a CNT in the momentum space representation is to use the zone-folding approximation [34]. The Hamiltonian of a CNT can be written in the sublattice basis for $A$ and $B$ sublattices,

$$
H_{0}=\sum_{\mathbf{k}, s} \gamma_{s}(\mathbf{k}) a_{\mathbf{k} s}^{\dagger} b_{\mathbf{k} s}+\text { H.c., }
$$

where $a_{\mathbf{k} s}^{\dagger}\left(b_{\mathbf{k} s}^{\dagger}\right)$ creates an electron on $A(B)$ sublattice with momentum $\mathbf{k}$ and spin $s$. The factor determining the kinetic energy $\gamma(\mathbf{k})$ is defined as

$$
\gamma_{s}(\mathbf{k})=t_{s, 1} e^{i \mathbf{k} \cdot \mathbf{a}_{1}}+t_{s, 2} e^{i \mathbf{k} \cdot \mathbf{a}_{2}}+t_{s, 3}
$$

where $t_{s, i}$ is the spin-dependent hopping parameter, see Fig. 1(a), between an $A$ atom and its $i$ th neighbor, and $\mathbf{a}_{1}$ and $\mathbf{a}_{2}$ are the Bravais lattice vectors of the graphene lattice. The low-energy unperturbed CNT Hamiltonian $H_{\mathrm{CNT}}$ can be obtained by an expansion of Eq. (A1) around the Dirac points $\mathbf{k}=\boldsymbol{\kappa}+\tau \mathbf{K}$ [42] and a rotation from sublattice into conduction/valence band basis. In the following, we will assume that the chemical potential is in the conduction band, obtaining

$$
H_{\mathrm{CNT}}-\mu N=\sum_{k, \tau, s} \xi_{\tau s}(k) c_{k \tau s}^{\dagger} c_{k \tau s}
$$

where $\xi_{\tau s}(k)=\varepsilon_{\tau s}(k)-\mu, \varepsilon_{\tau s}(k)=\left|\gamma_{\tau s}(k)\right|$ is the CNT single-particle energy in the conduction band, $\mu$ the chemical potential and $c_{k \tau s}^{\dagger}|0\rangle=|k \tau s\rangle$ define the basis of Eq. (2). The curvature of the CNT's lattice results in both spin-dependent and spin-independent modifications, which can be represented as shifts in both transverse and longitudinal momentum. Thus, the single-particle energies of a CNT in Eq. (2) for given transverse momentum $k_{\perp}$ and longitudinal momentum $k$ at low energies are given by

$$
\begin{aligned}
\varepsilon_{\tau s}\left(k_{\perp}, k\right)= & \hbar v_{F}\left\{\left(k-\tau K_{\|}+\tau \Delta k_{\|}^{c}\right)^{2}\right. \\
& \left.+\left(k_{\perp}-\tau K_{\perp}+\tau \Delta k_{\perp}^{c}+s \Delta k_{\perp}^{\mathrm{SO}}\right)^{2}\right\}^{1 / 2},
\end{aligned}
$$

where $K_{\perp}, K_{\|}$are the transverse and longitudinal components of momentum at the Dirac point $K$. The quantum numbers $\tau$ and $s$ are are defined in the main text. In the case of the $(12,4)$ semiconducting nanotube, the numerical values of those momentum shifts in our calculations are $\Delta k_{\perp}^{c}=-22.83 \mu \mathrm{m}^{-1}$, $\Delta k_{\|}^{c}=66.62 \mu \mathrm{m}^{-1}, \Delta k_{\mathrm{SO}}=-2.917 \mu \mathrm{m}^{-1}, K_{\|}=0$, and the lowest energy subbands shown in Fig. 1(b) have $k_{\perp}-\tau K_{\perp}=$ $\tau / 3 R$. The value of $k_{\perp}$ for the $K$ valley subband in our nanotube is $-35 / R$, where $R$ is the CNT radius. Note that the 
single-particle energies satisfy the time-reversal conjugation, $\varepsilon_{\tau s}(k)=\varepsilon_{-\tau-s}(-k)$.

The low-energy Bloch Hamiltonian Eq. (2) also contains the valley mixing and Zeeman field contributions, $H=$ $H_{\mathrm{CNT}}+H_{\Delta_{K K^{\prime}}}+H_{Z}$. Since the nanotube we are studying is of the zigzag class [36,37], to mix the valleys it is enough to break only the rotational symmetry. In our setup, we consider the valley mixing introduced by the presence of the substrate, modeling it as an electrostatic potential with a Gaussian distribution in the polar coordinate, $V(\varphi)=V_{0} \exp (-(\varphi-$ $\pi / 2) / \Delta \varphi)$. This corresponds to the substrate extending in the $x z$ plane. In the reciprocal space, the valley-mixing term is given by

$$
H_{K K^{\prime}}=\sum_{k, s} \Delta_{K K^{\prime}}\left(c_{k K s}^{\dagger} c_{k K^{\prime} s}+c_{k K^{\prime} s}^{\dagger} c_{k K s}\right)
$$

and couples states with the same spin and $k$ but opposite valley. Following Ref. [29], we set $\Delta_{K K^{\prime}}=2.5 \mathrm{meV}$.

The Zeeman effect with the field applied along the $x$ axis couples opposite spins in the same valley:

$$
H_{Z}=\sum_{k, \tau} \mu_{B} B_{\perp}\left(c_{k \tau \uparrow}^{\dagger} c_{k \tau \downarrow}+c_{k \tau \downarrow}^{\dagger} c_{k \tau \uparrow}\right) .
$$

The CNT Hamiltonian in Eq. (2) can be brought to a diagonal form by employing two unitary transformations. More details about the transformations can be found in Appendix D.1 of Ref. [29]. The first transformation diagonalizes the Hamiltonian without Zeeman energy $\left(B_{\perp}=0\right)$ and is defined as

$$
\left(\begin{array}{c}
c_{k K s} \\
c_{k K^{\prime} s}
\end{array}\right)=\left(\begin{array}{cc}
a_{s}(k) & b_{s}(k) \\
-b_{s}(k) & a_{s}(k)
\end{array}\right)\left(\begin{array}{c}
\alpha_{k s} \\
\beta_{k s}
\end{array}\right)
$$

with $a_{s}(k)^{2}+b_{s}(k)^{2}=1$ and the following values of $a_{s}(k)$ and $b_{s}(k)$ :

$$
\begin{aligned}
& a_{s}^{2}(k)=\frac{1}{2}\left(1-\frac{\xi_{K s}(k)-\xi_{K^{\prime} s}(k)}{\sqrt{\left(\xi_{K s}(k)-\xi_{K^{\prime} s}(k)\right)^{2}+4 \Delta_{K K^{\prime}}^{2}}}\right), \\
& b_{s}^{2}(k)=\frac{1}{2}\left(1+\frac{\xi_{K s}(k)-\xi_{K^{\prime} s}(k)}{\sqrt{\left(\xi_{K s}(k)-\xi_{K^{\prime} s}(k s)\right)^{2}+4 \Delta_{K K^{\prime}}^{2}}}\right),
\end{aligned}
$$

where the energy eigenvalues are

$$
\begin{aligned}
E_{ \pm s}(k)=\frac{1}{2}\left(\xi_{K s}(k)+\xi_{K^{\prime} s}(k)\right) & \\
& \pm \frac{1}{2} \sqrt{\left(\xi_{K s}(k)-\xi_{K^{\prime} s}(k)\right)^{2}+4 \Delta_{K K^{\prime}}^{2}}
\end{aligned}
$$

Due to the time-reversal conjugation of $\xi_{\tau s}(k)=\xi_{-\tau-s}(-k)$, it can be shown that $a_{s}(k)=b_{-s}(-k)$ and $E_{ \pm s}(k)=E_{ \pm-s}(-k)$.

Using Eq. (A7), the Zeeman term can be expressed as

$$
\begin{aligned}
& \tilde{B}_{\perp}=B_{\perp}\left(\left|a_{\uparrow}(k)\right|\left|a_{\downarrow}(k)\right|+\left|b_{\uparrow}(k)\right|\left|b_{\downarrow}(k)\right|\right), \\
& B_{\perp}^{\star}=B_{\perp}\left(\left|a_{\uparrow}(k)\right|\left|b_{\downarrow}(k)\right|-\left|b_{\uparrow}(k)\right|\left|a_{\downarrow}(k)\right|\right) .
\end{aligned}
$$

The magnetic field $\tilde{B}_{\perp}$ couples the spins within the lower and upper band pair, while $B_{\perp}^{\star}$ couples the spins between band pairs. Both are symmetric in $k$, i.e., $\tilde{B}_{\perp}(k)=\tilde{B}_{\perp}(-k)$ and $B_{\perp}^{\star}(k)=B_{\perp}^{\star}(-k)$. This is a consequence of the pseudo-timereversal symmetry.
In the regime of small Zeeman energy, i.e., $\Delta E=$ $\left|E_{+s}-E_{-s}\right|>\mu_{B} B_{\perp}$, the terms with $B_{\perp}^{\star}$ can be omitted. This allows us to treat the upper and lower pair of bands separately. We shall proceed to find the solutions for the lower band pair only, assuming that the chemical potential $\mu$ is tuned into the gap between the two energy bands $\tilde{E}_{1}$ and $\tilde{E}_{2}$. Therefore, we will neglect the influence of the bands $\tilde{E}_{3}$ and $\tilde{E}_{4}$ because those bands are not occupied. Then, the second transformation diagonalizing the Hamiltonian with magnetic field is defined as

$$
\left(\begin{array}{c}
\alpha_{k \uparrow} \\
\alpha_{k \downarrow}
\end{array}\right)=\left(\begin{array}{cc}
g(k) & h(k) \\
-h(k) & g(k)
\end{array}\right)\left(\begin{array}{l}
f_{k 1} \\
f_{k 2}
\end{array}\right)
$$

where the coefficients must satisfy $g^{2}(h)+h^{2}(k)=1$. The new quantum number in Eq. (A12) $i \in\{1,2\}$ just reflects the ordering of the energy bands $E_{1}<E_{2}$. The coefficients $g$ and $h$ are defined as

$$
\begin{aligned}
& g^{2}(k)=\frac{1}{2}\left(1-\frac{E_{-\uparrow}(k)-E_{-\downarrow}(k)}{\sqrt{\left(E_{-\uparrow}(k)-E_{-\downarrow}(k)\right)^{2}+4\left(\mu_{B} \tilde{B}_{\perp}\right)^{2}}}\right), \\
& h^{2}(k)=\frac{1}{2}\left(1+\frac{E_{-\uparrow}(k)-E_{-\downarrow}(k)}{\sqrt{\left(E_{-\uparrow}(k)-E_{-\downarrow}(k)\right)^{2}+4\left(\mu_{B} \tilde{B}_{\perp}\right)^{2}}}\right) .
\end{aligned}
$$

The coefficients satisfy the pseudo-time-reversal conjugation $g(k)=h(-k)$. Then, the single-particle energies of the full Hamiltonian with decoupled band pairs are

$$
\begin{aligned}
\tilde{E}_{i}(k)= & \frac{1}{2}\left(E_{-\uparrow}(k)+E_{-\downarrow}(k)\right) \\
& +(-1)^{i} \frac{1}{2} \sqrt{\left(E_{-\uparrow}(k)-E_{-\downarrow}(k)\right)^{2}+4\left(\mu_{B} \tilde{B}_{\perp}\right)^{2}} .
\end{aligned}
$$

The renormalized magnetic field opens a band gap at the $\Gamma$ point. The single-particle energies have the property $\tilde{E}_{i}(k)=\tilde{E}_{i}(-k)$ with $i \in\{1,2\}$ because $\tilde{B}_{\perp}(k)=\tilde{B}_{\perp}(-k)$. This pseudo-time-reversal symmetry for conduction band states results in the relation depicted in Fig. 1(f). Since the single-particle states of a finite CNT in our setup contain both $\vec{k}, s$ and $-\vec{k},-s$ contributions with equal weights, their spin components in the real space must also obey the relation shown in Fig. 1(e).

\section{Pseudo-time-reversal symmetry}

The pseudo-time-reversal invariance holds exactly for our effective model Hamiltonian Eq. (2). For the real space Hamiltonian, given by Eq. (1) in Ref. [29], with hopping terms given by Eq. (9) in Ref. [42], it is, however, broken by two effects, both absent in our four-band model. The first and smaller one is the presence of nearest-neighbor hoppings with spin flip, which couple neighboring angular momentum subbands $[30,32,42]$.

The second and dominant effect is due to the modification of the hoppings by the magnetic field [47] which destroys 

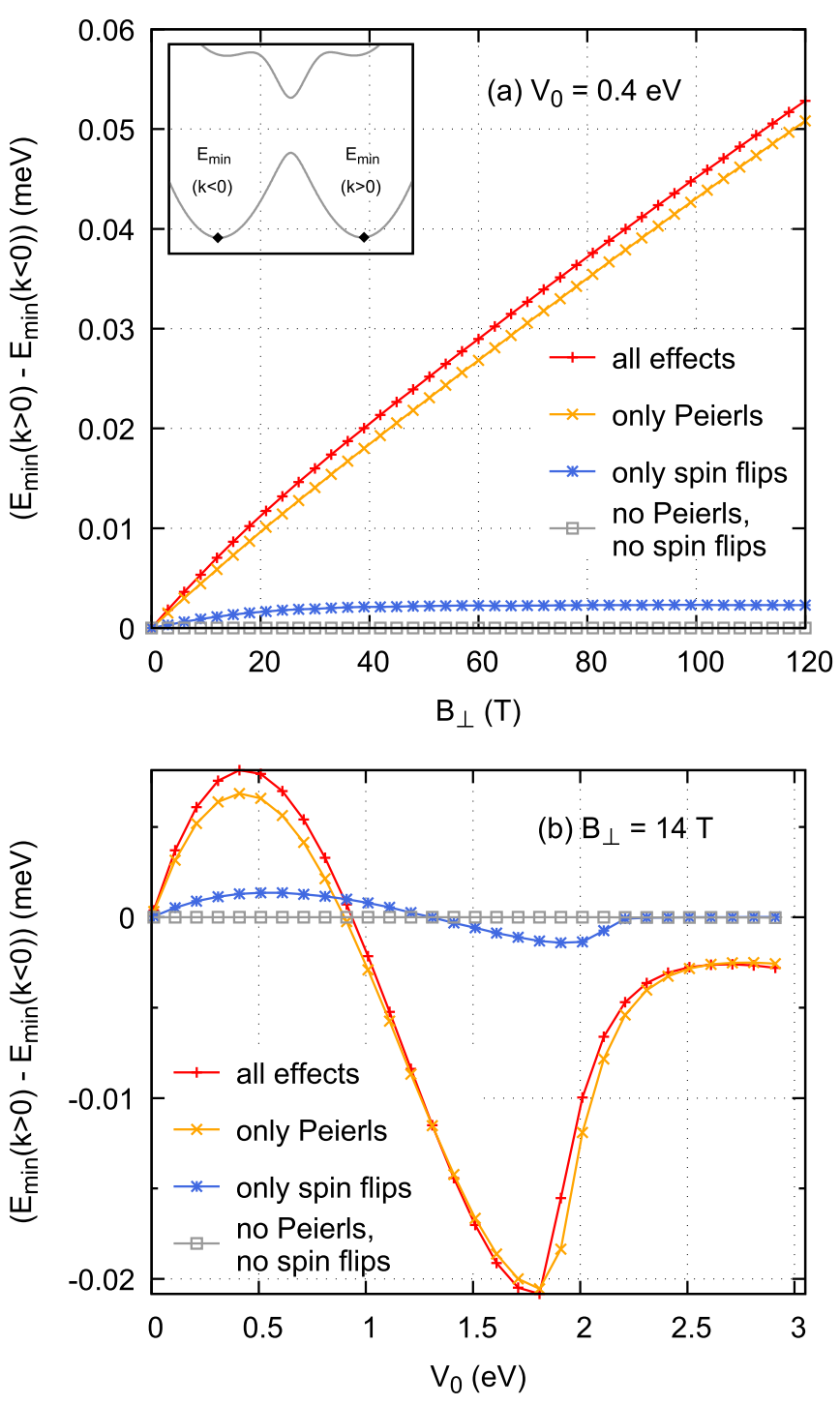

FIG. 7. The breaking of $\tilde{\mathcal{T}}$ as a function of $B_{\perp}$ and of $V_{0}$. (a) The substrate potential has the amplitude $V_{0}=0.4 \mathrm{eV}$, the value taken in the simulations shown in the main text. (b) The magnetic field is $B_{\perp}=14 \mathrm{~T}$ as in the main text and the strength $V_{0}$ of the substrate potential is varied.

$\tilde{\mathcal{T}}$ by adding the same complex phase (Peierls phase) to all hoppings. Figure 7 shows the strength of $\tilde{\mathcal{T}}$ breaking, quantified as the difference between the band (1) (cf. Fig. 1) minima at $k>0$ and at $k<0$, as a function of $B_{\perp}$ and of $V_{0}$, as shown in the inset of Fig. 7(a). The contribution of the spin-flipping terms is an order of magnitude smaller than that of the Peierls phase. This can be understood by noticing that the Peierls phase affects the terms with largest energy scales, i.e., the hopping integrals of the order of $\sim \mathrm{eV}$. The spin-flipping term is comparatively weak, acting only on a $\mathrm{meV}$ scale. Omitting both terms in the Hamiltonian restores the symmetry between $k<0$ and $k>0$.

Nevertheless, even when both effects are present, for our parameters $B_{\perp}=14 \mathrm{~T}$ and $V_{0}=0.4 \mathrm{eV}$ the pseudo-timereversal still holds down to $\mu \mathrm{eV}$ energy scales.

\section{Superconducting spectrum}

Including superconducting correlations on a mean-field level, we add to the Hamiltonian a superconducting pairing term [39], which is given by

$$
H_{\mathrm{SC}}=\sum_{k} \Delta_{0}\left(c_{k K \uparrow}^{\dagger} c_{-k K^{\prime} \downarrow}+c_{k K^{\prime} \uparrow}^{\dagger} c_{-k K \downarrow}+\text { H.c. }\right),
$$

where $\Delta_{0}$ is the superconducting order parameter, which we take to be $0.4 \mathrm{meV}$. We can express the pairing Hamiltonian Eq. (A15) in the eigenbasis of the CNT Eq. (2) and, after applying the approximations and transformations described in Appendix A 1, we obtain the BdG Hamiltonian Eq. (3) with the pairing terms:

$$
\begin{gathered}
\tilde{\Delta}_{p}(k)=\Delta_{0}\left(g^{2}(k)-h^{2}(k)\right)=-\tilde{\Delta}_{p}(-k), \\
\tilde{\Delta}_{s}(k)=2 \Delta_{0} g(k) h(k)=\tilde{\Delta}_{s}(-k) .
\end{gathered}
$$

We see that the pairing term $\tilde{\Delta}_{s}(k)$ has an even and $\tilde{\Delta}_{p}(k)$ an odd parity, as shown in Fig. 1(c).

The basis change which transforms Eq. (3) into Eq. (4) is given by

$$
\left(\begin{array}{c}
f_{k 1} \\
f_{-k 2}^{\dagger}
\end{array}\right)=\left(\begin{array}{cc}
m(k) & n(k) \\
-n(k) & m(k)
\end{array}\right)\left(\begin{array}{c}
d_{k+} \\
d_{-k-}^{\dagger}
\end{array}\right)
$$

with the normalization condition $m^{2}(k)+n^{2}(k)=1$ and the coefficients defined in the following way:

$$
\begin{aligned}
& m^{2}(k)=\frac{1}{2}\left(1+\frac{E_{1}(k)+E_{2}(k)}{\sqrt{\left(E_{1}(k)+E_{2}(k)\right)^{2}+\left(2 \tilde{\Delta}_{s}(k)\right)^{2}}}\right), \\
& n^{2}(k)=\frac{1}{2}\left(1-\frac{E_{1}(k)+E_{2}(k)}{\sqrt{\left(E_{1}(k)+E_{2}(k)\right)^{2}+\left(2 \tilde{\Delta}_{s}(k)\right)^{2}}}\right) .
\end{aligned}
$$

\section{APPENDIX B: 1D MAJORANA BOUND STATE SOLUTIONS}

MBSs are zero energy eigenstates of the BdG Hamiltonian and the particle-hole symmetry operator. The low-energy physics of the BdG Hamiltonian Eq. (4) is described by the block $\hat{\mathcal{H}}_{\mathrm{BdG}}^{+}$. For the MBSs, we will approximate the BdG Hamiltonian by $\hat{\mathcal{H}}_{\mathrm{BdG}}^{+} \approx \hat{\mathcal{H}}_{\mathrm{BdG}}^{\Gamma}+\hat{\mathcal{H}}_{\mathrm{BdG}}^{R}+\hat{\mathcal{H}}_{\mathrm{BdG}}^{L}$ because the low-energy physics of $\hat{\mathcal{H}}_{\mathrm{BdG}}^{+}$has three contributions, as illustrated in Fig. 3(a). This is also supported by the numerics, see Fig. 3(b).

\section{1. $\Gamma$-point contribution}

The first contribution is coming from the $\Gamma$ point. Therefore, we obtain from a Taylor expansion around the $\Gamma$ point

$$
\begin{aligned}
& \tilde{\xi}_{+}(k) \approx \tilde{\xi}_{+}(0)+\frac{\hbar^{2} k^{2}}{2 m^{\star}}, \\
& \tilde{\Delta}_{p}(k) \approx \lambda \hbar k,
\end{aligned}
$$


where $\frac{1}{m^{\star}}=\left.\frac{\partial^{2} \tilde{\xi}_{+}(k)}{\hbar^{2} \partial k^{2}}\right|_{k=0}$ and $\lambda=\left.\frac{\partial \tilde{\Delta}_{p}(k)}{\hbar \partial k}\right|_{k=0}$. Then the BdG Hamiltonian for $k \approx \Gamma$ becomes

$$
\hat{\mathcal{H}}_{\mathrm{BdG}}^{\Gamma}=\left(\begin{array}{cc}
\frac{\hbar^{2} k^{2}}{2 m^{\star}}+\tilde{\xi}_{+}(0) & \lambda \hbar k \\
\lambda \hbar k & -\left(\frac{\hbar^{2} k^{2}}{2 m^{\star}}+\tilde{\xi}_{+}(0)\right)
\end{array}\right),
$$

and the corresponding $\mathrm{BdG}$ equation reads

$$
\left(\begin{array}{cc}
\frac{\hbar^{2} k^{2}}{2 m^{\star}}+\tilde{\xi}_{+}(0) & \lambda \hbar k \\
\lambda \hbar k & -\left(\frac{\hbar^{2} k^{2}}{2 m^{\star}}+\tilde{\xi}_{+}(0)\right)
\end{array}\right)\left(\begin{array}{l}
u_{\Gamma} \\
v_{\Gamma}
\end{array}\right)=E\left(\begin{array}{l}
u_{\Gamma} \\
v_{\Gamma}
\end{array}\right) .
$$

Now, we interpret $k$ as the momentum operator $k \rightarrow \hat{k}=$ $-i \partial_{z}$ and make the ansatz

$$
\left(\begin{array}{l}
u_{\Gamma}(z) \\
v_{\Gamma}(z)
\end{array}\right)=\left(\begin{array}{l}
u_{\Gamma} \\
v_{\Gamma}
\end{array}\right) e^{\kappa_{\Gamma} z}
$$

For the momentum $\kappa_{\Gamma}$, we need to solve the secular equation $\operatorname{det}\left(\hat{\mathcal{H}}_{\mathrm{BdG}}^{\Gamma}-E \mathbb{1}\right) \stackrel{!}{=} 0$ for any energy $E$ and we obtain

$$
\begin{aligned}
\kappa_{\Gamma}^{2}= & 2 \frac{m^{\star} \tilde{\xi}_{+}(0)}{\hbar^{2}}+2\left(\frac{m^{\star} \lambda}{\hbar}\right)^{2} \\
& \pm \sqrt{\left(2 \frac{m^{\star} E}{\hbar^{2}}\right)^{2}+4\left(\frac{m^{\star} \lambda}{\hbar}\right)^{2}\left(\left(\frac{m^{\star} \lambda}{\hbar}\right)^{2}+2 \frac{m^{\star} \tilde{\xi}_{+}(0)}{\hbar^{2}}\right)} .
\end{aligned}
$$

For zero-energy modes, the equation can be simplified:

$$
\kappa_{\Gamma}= \pm\left(\frac{m^{\star} \lambda}{\hbar} \pm \sqrt{\left(\frac{m^{\star} \lambda}{\hbar}\right)^{2}+2 \frac{m^{\star} \tilde{\xi}_{+}(0)}{\hbar^{2}}}\right) .
$$

The corresponding zero energy eigenvectors are given by

$$
\left(\begin{array}{c}
u_{\Gamma} \\
v_{\Gamma}
\end{array}\right)=\frac{1}{\sqrt{2}}\left(\begin{array}{c}
\mp i \\
1
\end{array}\right)
$$

\section{Fermi point contribution}

For the Fermi point contribution, we need to linearize $\tilde{\xi}_{+}(k)$ around $k_{F}$ and $-k_{F}$, see Fig. 3. Then, we can define the following two Nambu spinors $\Psi_{R}=\left(d_{k+, R}, d_{-k+, L}^{\dagger}\right)$ and $\Psi_{L}=\left(d_{k+, L}, d_{-k+, R}^{\dagger}\right)$. The subscripts $R, L$ denote the right and left movers. The corresponding BdG Hamiltonians are given by

$$
\begin{aligned}
& \hat{\mathcal{H}}_{\mathrm{BdG}}^{R}=\left(\begin{array}{cc}
v_{F} \hbar\left(k-k_{F}\right) & \tilde{\Delta}_{p}\left(k_{F}\right) \\
\tilde{\Delta}_{p}\left(k_{F}\right) & -v_{F} \hbar\left(k-k_{F}\right)
\end{array}\right), \\
& \hat{\mathcal{H}}_{\mathrm{BdG}}^{L}=\left(\begin{array}{cc}
-v_{F} \hbar\left(k+k_{F}\right) & \tilde{\Delta}_{p}\left(-k_{F}\right) \\
\tilde{\Delta}_{p}\left(-k_{F}\right) & v_{F} \hbar\left(k+k_{F}\right)
\end{array}\right),
\end{aligned}
$$

where for $\hat{\mathcal{H}}_{\mathrm{BdG}}^{R}$ we have $k>0$ and for $\hat{\mathcal{H}}_{\mathrm{BdG}}^{L}$ we have $k<0$. The corresponding BdG equation reads

$$
\begin{aligned}
& \left(\begin{array}{cc}
v_{F} \hbar\left(k-k_{F}\right) & \tilde{\Delta}_{p}\left(k_{F}\right) \\
\tilde{\Delta}_{p}\left(k_{F}\right) & -v_{F} \hbar\left(k-k_{F}\right)
\end{array}\right)\left(\begin{array}{l}
u_{R} \\
v_{L}
\end{array}\right)=E\left(\begin{array}{l}
u_{R} \\
v_{L}
\end{array}\right), \\
& \left(\begin{array}{cc}
-v_{F} \hbar\left(k+k_{F}\right) & \tilde{\Delta}_{p}\left(-k_{F}\right) \\
\tilde{\Delta}_{p}\left(-k_{F}\right) & v_{F} \hbar\left(k+k_{F}\right)
\end{array}\right)\left(\begin{array}{l}
u_{L} \\
v_{R}
\end{array}\right)=E\left(\begin{array}{l}
u_{L} \\
v_{R}
\end{array}\right) .
\end{aligned}
$$

With $k \rightarrow \hat{k}=-i \partial_{z}$ and making the ansatz

$$
\left(\begin{array}{l}
u_{R}(z) \\
v_{L}(z)
\end{array}\right)=\left(\begin{array}{l}
u_{R} \\
v_{L}
\end{array}\right) e^{\kappa_{R} z} \text { and }\left(\begin{array}{c}
u_{L}(z) \\
v_{R}(z)
\end{array}\right)=\left(\begin{array}{c}
u_{L} \\
v_{R}
\end{array}\right) e^{\kappa_{L} z},
$$

we get the decay lengths $\kappa_{R}$ and $\kappa_{L}$ from the secular equations $\operatorname{det}\left(\hat{\mathcal{H}}_{\mathrm{BdG}}^{\mathrm{R} / \mathrm{L}}-E \mathbb{1}\right) \stackrel{!}{=} 0$. The decay lengths for the zero-energy modes become $\kappa_{R}=i k_{F} \mp \frac{\left|\tilde{\Delta}_{p}\left(k_{F}\right)\right|}{v_{F} \hbar}$ and $\kappa_{L}=-i k_{F} \mp \frac{\left|\tilde{\Delta}_{p}\left(k_{F}\right)\right|}{v_{F} \hbar}$. Furthermore, we get the two eigenvectors

$$
\begin{aligned}
& \left(\begin{array}{l}
u_{R} \\
v_{L}
\end{array}\right)=\frac{1}{\sqrt{2}}\left(\begin{array}{c} 
\pm i \operatorname{sign}\left(\tilde{\Delta}_{p}\left(k_{F}\right)\right) \\
1
\end{array}\right)=\frac{1}{\sqrt{2}}\left(\begin{array}{c}
\mp i \\
1
\end{array}\right), \\
& \left(\begin{array}{l}
u_{L} \\
v_{R}
\end{array}\right)=\frac{1}{\sqrt{2}}\left(\begin{array}{c}
\mp i \operatorname{sinn}\left(\tilde{\Delta}_{p}\left(-k_{F}\right)\right) \\
1
\end{array}\right)=\frac{1}{\sqrt{2}}\left(\begin{array}{c}
\mp i \\
1
\end{array}\right),
\end{aligned}
$$

where we used $\operatorname{sgn}\left(\tilde{\Delta}_{p}\left(k_{F}\right)\right)=-1$ and $\operatorname{sgn}\left(\tilde{\Delta}_{p}\left(-k_{F}\right)\right)=+1$, see Fig. 1(b).

\section{APPENDIX C: CONSTRUCTION OF 3D MAJORANA WAVE FUNCTION}

Explicitly, the coefficients of the electron and holes are parts of the MBS Eq. (9) given by

$$
\begin{aligned}
u_{K \uparrow}(k) & =a_{\uparrow}(k)[u m(k) g(k)-v n(k) h(k)], \\
u_{K^{\prime} \uparrow}(k) & =-b_{\uparrow}(k)[u m(k) g(k)-v n(k) h(k)], \\
v_{K \uparrow}(k) & =a_{\uparrow}(k)[v m(k) g(k)-u n(k) h(k)], \\
v_{K^{\prime} \uparrow}(k) & =-b_{\uparrow}(k)[v m(k) g(k)-u n(k) h(k)], \\
u_{K \downarrow}(k) & =-a_{\downarrow}(k)[u m(k) h(k)+v n(k) g(k)], \\
u_{K^{\prime} \downarrow}(k) & =b_{\downarrow}(k)[u m(k) h(k)+v n(k) g(k)], \\
v_{K_{\downarrow}}(k) & =-a_{\downarrow}(k)[v m(k) h(k)+u n(k) g(k)], \\
v_{K^{\prime} \downarrow}(k) & =b_{\downarrow}(k)[v m(k) h(k)+u n(k) g(k)] .
\end{aligned}
$$

\section{APPENDIX D: EFFECTS OF LOCAL PROXIMITY}

The isotropic superconducting pairing introduced in the main text has a heuristic justification in the large size of the proximity length in the usual superconductor/metal junctions, of the order of 10-100 nm [49], much larger than the CNT diameter. Nevertheless, the reduced dimensionality of the CNT offers only a small area for tunneling between the nanotube and the superconducting substrate. We model this with a superconducting pairing amplitude which decays exponentially with the distance from the line of contact between the CNT and the superconductor,

$$
\Delta(\varphi)=\Delta_{0} \exp \left(-\frac{R \cos \left(\varphi-\varphi_{0}\right)}{\lambda_{\Delta}}\right)
$$

where $\varphi_{0}$ is the polar angle of the contact line and $\lambda_{\Delta}$ is a characteristic length for the decay of the proximity effect. We find that the superconducting gap is still present in the CNT and can be closed by the application of $B_{\perp}$, as shown in Fig. 8(a). The size of the effective superconducting gap decreases with increasing $\lambda_{\Delta}$, as can be seen in the neighborhood of the $k_{\|}= \pm 0.1 \mathrm{~nm}^{-1}$ points, where the gap is not affected by the Zeeman field. Since the gap is smaller, a lower magnetic field suffices to close it. The ranges of polar angles 


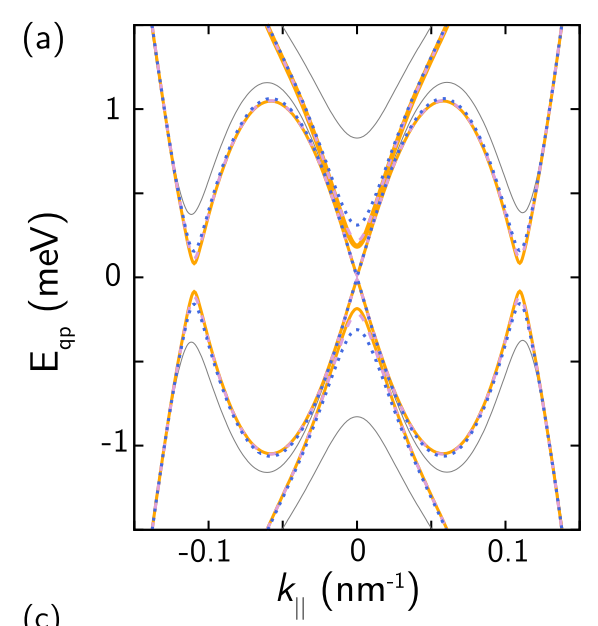

(c)

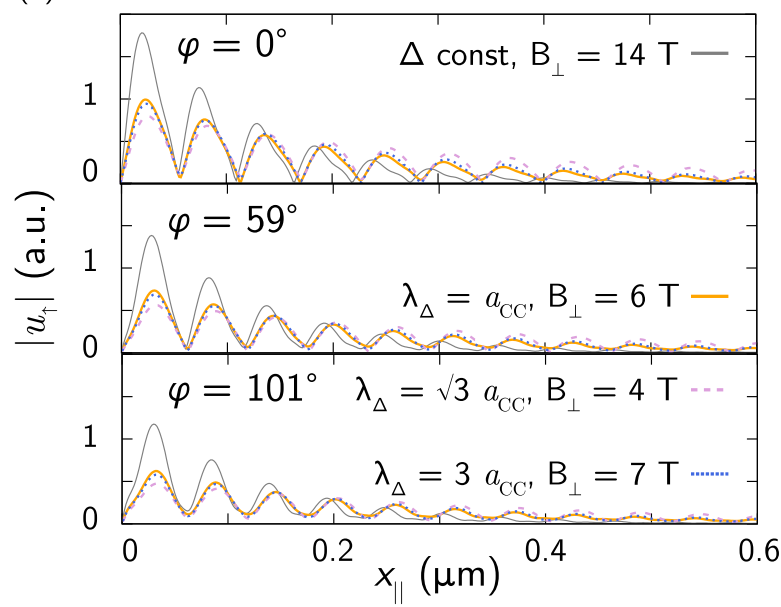

(e)

(b)

$-\lambda_{\Delta}=a_{\mathrm{CC}}$ $\left(\mathrm{B}_{\perp}=8.5 \mathrm{~T}, \Delta_{0}=0.4 \mathrm{meV}\right)$

$\left(\mathrm{B}_{\perp}=2 \mathrm{~T}\right.$,

$\Delta_{0}=0.8 \mathrm{meV}$ )

$=-\lambda_{\Delta}=\sqrt{3} a_{\mathrm{CC}}$

$\left(\mathrm{B}_{\perp}=2 \mathrm{~T}\right.$,

$\left.\Delta_{0}=0.4 \mathrm{meV}\right)$

$\lambda_{\Delta}=3 a_{\mathrm{CC}}$

$\left(\mathrm{B}_{\perp}=3 \mathrm{~T}, \Delta_{0}=0.4 \mathrm{meV}\right)$

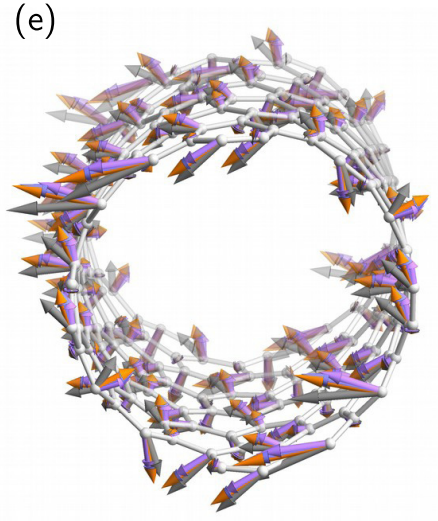

(d)

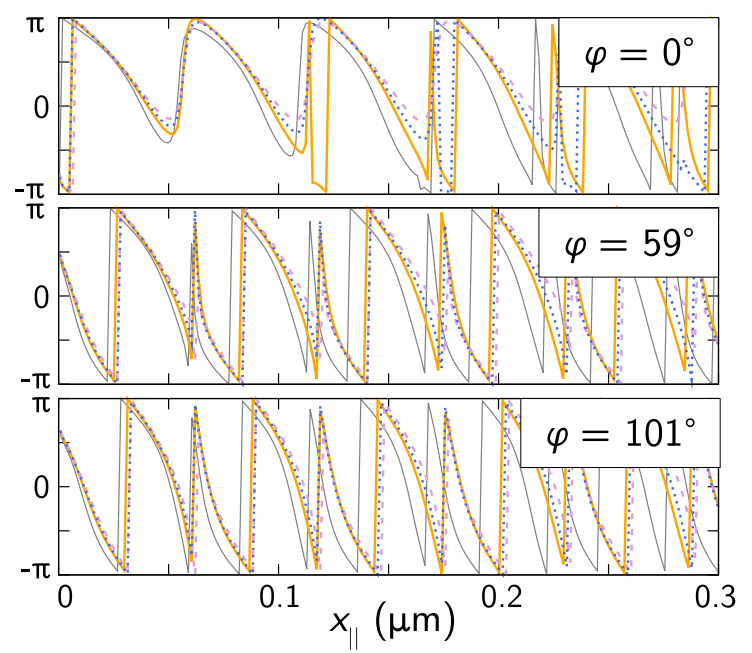

FIG. 8. The influence of a localized superconducting pairing on the bulk spectrum and the wave function of the Majorana states. (a) For different values of the parameter $\lambda_{\Delta}$, the band gap still closes, but at different values of $B_{\perp}$. For $\lambda_{\Delta}=a_{\mathrm{CC}}$ (the carbon-carbon bond length), we set $\Delta_{0}=0.8 \mathrm{meV}$ to keep a clear band gap. (b) The polar angle range with strongest pairing, i.e., $R \cos \left(\varphi-\varphi_{0}\right)<\lambda_{\Delta}$; the grey circle represents constant amplitude over the CNT circumference, the arc fragments are color coded as in (a). Cuts across $\left|u_{i \uparrow}\right|$ (c) and the spin canting angle (d) along several values of the polar angle $\varphi$. The corresponding values of $B_{\perp}$ are the same in (c) and (d). (e) The local spin direction of the particle component of the Majorana spinor; the arrows representing electron spin for various values of $\lambda_{\Delta}$ are superposed on each atom to show that the spin direction is not much influenced by $\lambda_{\Delta}$. The color code is the same as in (a)-(d), $B_{\perp}$ for each $\lambda_{\Delta}$ is the same as in (c), (d).

where the atoms are within $\lambda_{\Delta}$ distance from the substrate are shown in Fig. 8(b). With reduced gap size, the Majorana states forming in the topological phase decay more slowly, but both the oscillations of the wave function amplitude displayed in Fig. 8(c) and the spin-canting angle in Fig. 8(d) remain qualitatively the same as for $\Delta=$ const $=\Delta_{0}$. A close look at the spin expectation value in the particle part of the Majorana spinor at the very left end of the CNT [cf. Fig. 8(e)] shows that the spin texture at the left end does not vary strongly with $\lambda_{\Delta}$ and still many spin directions are present.
[1] R. Aguado, La Rivista del Nuovo Cimento 40, 523 (2017).

[2] A. Y. Kitaev, Phys. Usp. 44, 131 (2001).

[3] M. Sato and Y. Ando, Rep. Prog. Phys. 80, 076501 (2017).

[4] R. M. Lutchyn, J. D. Sau, and S. Das Sarma, Phys. Rev. Lett. 105, 077001 (2010).

[5] Y. Oreg, G. Refael, and F. von Oppen, Phys. Rev. Lett. 105, 177002 (2010).

[6] R. M. Lutchyn, E. P. A. M. Bakkers, L. P. Kouwenhoven, P. Krogstrup, C. M. Marcus, and Y. Oreg, Nat. Rev. Mater. 3, 52 (2018).
[7] V. Mourik, K. Zuo, S. M. Frolov, S. R. Plissard, E. P. A. M. Bakkers, and L. P. Kouwenhoven, Science 336, 1003 (2012).

[8] H. O. H. Churchill, V. Fatemi, K. Grove-Rasmussen, M. T. Deng, P. Caroff, H. Q. Xu, and C. M. Marcus, Phys. Rev. B 87, 241401(R) (2013).

[9] M. T. Deng, S. Vaitiekenas, E. B. Hansen, J. Danon, M. Leijnse, K. Flensberg, J. Nygård, P. Krogstrup, and C. M. Marcus, Science 354, 1557 (2016).

[10] H. Zhang, Ö. Gül, S. Conesa-Boj, M. P. Nowak, M. Wimmer, K. Zuo, V. Mourik, F. K. de Vries, J. van Veen, 
M. W. A. de Moor, J. D. S. Bommer, D. J. van Woerkom, D. Car, S. R. Plissard, E. P. A. M. Bakkers, M. Quintero-Pérez, M. C. Cassidy, S. Koelling, S. Goswami, K. Watanabe, T. Taniguchi, and L. P. Kouwenhoven, Nat. Commun. 8, 16025 (2017).

[11] C.-X. Liu, J. D. Sau, T. D. Stanescu, and S. Das Sarma, Phys. Rev. B 96, 075161 (2017).

[12] E. Prada, R. Aguado, and P. San-Jose, Phys. Rev. B 96, 085418 (2017).

[13] D. J. Clarke, Phys. Rev. B 96, 201109(R) (2017).

[14] E. M. Spanton, M. Deng, S. Vaitiekénas, P. Krogstrup, J. Nygård, C. M. Marcus, and K. A. Moler, Nat. Phys. 13, 1177 (2017).

[15] S. Hoffman, D. Chevallier, D. Loss, and J. Klinovaja, Phys. Rev. B 96, 045440 (2017).

[16] A. Schuray, A. L. Yeyati, and P. Recher, Phys. Rev. B 98, 235301 (2018).

[17] D. Sticlet, C. Bena, and P. Simon, Phys. Rev. Lett. 108, 096802 (2012).

[18] N. Sedlmayr and C. Bena, Phys. Rev. B 92, 115115 (2015).

[19] J. Klinovaja and D. Loss, Phys. Rev. B 86, 085408 (2012).

[20] J. S. Lim, R. Lopez, and L. Serra, Europhys. Lett. 103, 37004 (2013).

[21] J. Osca, R. López, and L. Serra, Eur. Phys. J. B 87, 84 (2014).

[22] A. Manolescu, A. Sitek, J. Osca, L. Serra, V. Gudmundsson, and T. D. Stanescu, Phys. Rev. B 96, 125435 (2017).

[23] T. D. Stanescu, A. Sitek, and A. Manolescu, Beilstein J. Nanotechnol. 9, 1512 (2018).

[24] B. D. Woods, T. D. Stanescu, and S. Das Sarma, Phys. Rev. B 98, 035428 (2018).

[25] R. Egger and K. Flensberg, Phys. Rev. B 85, 235462 (2012).

[26] J. Klinovaja, S. Gangadharaiah, and D. Loss, Phys. Rev. Lett. 108, 196804 (2012).

[27] J. D. Sau and S. Tewari, Phys. Rev. B 88, 054503 (2013).

[28] C.-H. Hsu, P. Stano, J. Klinovaja, and D. Loss, Phys. Rev. B 92 , 235435 (2015).

[29] M. Marganska, L. Milz, W. Izumida, C. Strunk, and M. Grifoni, Phys. Rev. B 97, 075141 (2018).
[30] W. Izumida, K. Sato, and R. Saito, J. Phys. Soc. Jpn. 78, 074707 (2009).

[31] J. Klinovaja, M. J. Schmidt, B. Braunecker, and D. Loss, Phys. Rev. B 84, 085452 (2011).

[32] T. Ando, J. Phys. Soc. Jpn. 69, 1757 (2000).

[33] In our tight-binding model, we consider one $p_{z}$ orbital per atom.

[34] R. Saito, G. Dresselhaus, and M. S. Dresselhaus, Physical Properties of Carbon Nanotubes (Imperial College Press, London, 1998).

[35] F. Kuemmeth, S. Ilani, D. C. Ralph, and P. L. McEuen, Nature 452, 448 (2008).

[36] M. Marganska, P. Chudzinski, and M. Grifoni, Phys. Rev. B 92, 075433 (2015).

[37] W. Izumida, R. Okuyama, A. Yamakage, and R. Saito, Phys. Rev. B 93, 195442 (2016).

[38] X. Xi, Z. Wang, W. Zhao, J.-H. Park, K. T. Law, H. Berger, L. Forró, J. Shan, and K. F. Mak, Nat. Phys. 12, 139 (2016).

[39] B. Uchoa and A. H. Castro Neto, Phys. Rev. Lett. 98, 146801 (2007).

[40] K. Shiozaki and M. Sato, Phys. Rev. B 90, 165114 (2014).

[41] Y. Ando and L. Fu, Annu. Rev. Condens. Matter Phys. 6, 361 (2015).

[42] M. del Valle, M. Margańska, and M. Grifoni, Phys. Rev. B 84, 165427 (2011).

[43] S. Jeon, Y. Xie, J. Li, Z. Wang, B. A. Bernevig, and A. Yazdani, Science 358, 772 (2017).

[44] J. Cao, Q. Wang, and H. Dai, Nat. Mater. 4, 745 (2005).

[45] V. V. Deshpande, B. Chandra, R. Caldwell, D. S. Novikov, J. Hone, and M. Bockrath, Science 323, 106 (2009).

[46] M. Jung, J. Schindele, S. Nau, M. Weiss, A. Baumgartner, and C. Schönenberger, Nano Lett. 13, 4522 (2013).

[47] R. Peierls, Z. Phys. 80, 763 (1933).

[48] M. Marganska, Microscopic finder of energy levels and eigenstates of proximitized carbon nanotubes, https://doi.org/10. 5281/zenodo.3460775.

[49] M. Tinkham, Introduction to Superconductivity, 2nd ed. (Dover Publications Inc., New York, 2004). 